\title{
LPV Gain-Scheduled Observer-Based State Feedback for Active Control of Harmonic Disturbances with Time-Varying Frequencies
}

\author{
Wiebke Heins, Pablo Ballesteros, Xinyu Shu and Christian Bohn
}

Additional information is available at the end of the chapter

http://dx.doi.org/10.5772/50293

\section{Introduction}

The design of controllers for the rejection of multisine disturbances with time-varying frequencies is considered. The frequencies are assumed to be known. Such a control problem frequently arises in active noise and vibration control (ANC/AVC) applications where the disturbances are caused by imbalances due to rotating or oscillating masses or periodically fluctuating excitations, for example the torque of a combustion engine, and the rotational speed is measured. Application examples are automobiles and aircrafts.

For the rejection of disturbances with time-varying frequencies, time-varying controllers that are automatically adjusted to the disturbance frequencies are usually used. Although time-invariant controllers might be sufficient in some applications [22], time-varying controllers usually result in a much better performance, particularly if the disturbance frequencies vary over fairly wide ranges. Such a controller can be constructed in several ways (see Sec. 2.1). Two observer-based state-feedback controllers are presented in this chapter (see Sec. 3). General output-feedback controllers are treated in the next chapter. The approaches presented in this chapter use state augmentation in order to achieve disturbance rejection. One consists of a time-invariant plant observer and a time-varying state-feedback gain for the state-augmented plant, where the state augmenation is based on a time-varying error filter, as proposed by Kinney \& de Callafon [19]. The other controller approach is based on the disturbance observer of Bohn et al. [7], where the plant is augmented with a time-varying disturbance model. A time-varying observer for the overall system and a time-invariant state-feedback gain are used to track and reject the disturbance.

The remainder of this chapter is organized as follows. In Sec. 2, existing approaches to the problem are classified and some general control design considerations are discussed. The state-augmented observer-based state-feedback approaches are described in Sec. 3. In Sec. 4, 
the calculation of stabilizing state-feedback gains for time-varying systems in polytopic linear parameter-varying (pLPV) form is discussed. The application of this method for the rejection of harmonic disturbances is treated in Sec. 5. Real-time results are presented in Sec. 6. A discussion and conclusions are given in Sec. 7 .

\section{Controller design: Overview, stability and implementation aspects}

In this section, an overview of control approaches for the rejection of harmonic disturbances with time-varying frequencies is given (Sec. 2.1) and stability and implementation aspects are discussed (Secs. 2.2 and 2.3, respectively).

\subsection{Overview and classification of control approaches}

A common approach in ANC/AVC is adaptive filtering, where the filter weights are usually updated with the FxLMS algorithm [24]. In most cases, disturbance feedforward is used, although it is possible to use an adaptive filter in feedback control with a technique called "secondary path neutralization" [24], which is equivalent to internal model control [25].

Another approach is to use gain scheduling, where the scheduling parameters are calculated from the disturbance frequencies. This can be further subdivided into indirect and direct scheduling methods. In indirect scheduling, the controller, or part of it, for example a state-feedback or observer gain, is determined from a set of pre-computed quantities through interpolation or switching. For example, for linear parameter-varying (LPV) systems, where the uncertain parameters are contained in a polytope, one controller is calculated for each vertex and the resulting controller is obtained from interpolation [2]. For the rejection of harmonic disturbances, continuous-time LPV approaches have been suggested by Darengosse \& Chevrel [10], Du \& Shi [11], Du et al. [12], Witte et al. [28], Balini et al. [6] and tested for a single sinusoidal disturbance by Darengosse \& Chevrel [10], Du et al. [12], Witte et al. [28] and Balini et al. [6]. Methods based on observer-based state-feedback controllers are presented by Bohn et al. [7, 8], Kinney \& de Callafon [19, 20, 21] and Heins et al. [16, 17]. In the approach of Bohn et al. [7, 8], the observer gain is selected from a set of pre-computed gains by switching. In the other approaches of Kinney \& de Callafon [21], Heins et al. [17] and in this chapter, the observer gain is calculated by interpolation. In the other approach presented here, which is also used by Kinney \& de Callafon [19, 20] and Heins et al. [16], the state-feedback gain is scheduled using interpolation.

In direct scheduling, the dependence of the controller on the scheduling parameter does not correspond to a simple interpolation or switching law $[2,4,5,21,23,26]$. For example, for LPV systems where the parameter dependence is expressed as a linear fractional transformation (LFT), the uncertain parameters also enter the controller through an LFT [2]. For harmonic disturbances, an LPV-LFT approach has been suggested and applied in real time by Ballesteros \& Bohn [4, 5] and Shu et al. [26]. Another example for direct scheduling is a controller based on a time-varying state estimator, for example a Kalman filter, where the scheduling parameters enter the controller through the recursive equations for the state estimate and the error covariance matrix. Such a controller is presented and compared to an indirect (interpolation) approach by Kinney \& de Callafon [21]. 


\subsection{Stability considerations}

The existing design approaches can be classified as approaches that take stability into consideration and such that do not. In indirect scheduling, for example, the controllers or gains are sometimes pre-computed for fixed operating points and then interpolated in an ad-hoc fashion $[7,8,20]$. Stability is then not guaranteed, although it might be expected that the system is stable for slow variations of the scheduling parameter. For the adaptive filtering approaches, only approximate stability results seem available to date [13,24]. To take stability into consideration, it is attractive to model the control problem as an LPV system and then use suitable gain-scheduling techniques $[4-6,10,16,17,19,23,26,28]$. If parameter-independent Lyapunov functions are used, this guarantees closed-loop stability even for arbitrarily fast changes of the scheduling parameters at the expense of conservatism. The methods presented in this chapter guarantee closed-loop stability. In designs based on parameter-dependent Lyapunov functions, limits on the rate of change of the parameters can be incorporated.

\subsection{Implementation aspects}

For a practical application, the resulting controller has to be implemented in discrete time. In applications of ANC/AVC, the plant model is often obtained through system identification. This usually gives a discrete-time plant model. It is therefore most natural to carry out the whole design in discrete time. If a continuous-time controller is computed, the controller has to be discretized. Since the controllers considered here are time-varying, the discretization would have to be carried out at each sampling instant. An exact discretization involves the calculation of a matrix exponential, which is computationally too expensive. Particularly in LPV gain-scheduling control, an approximate discretization is proposed by Apkarian [3]. However, this leads to a distortion of the frequency scale. Usually, this can be tolerated, but not for the suppression of harmonic disturbances. It is therefore not surprising that the continuous-time controllers of Darengosse \& Chevrel [10], Du et al. [12], Kinney \& de Callafon [19] and Köroğlu \& Scherer [23] are only tested in simulation studies with a very simple system as a plant and a single frequency in the disturbance signal. The design methods that are tested in real time are usually formulated in discrete time $[4,5,7,8,16,17,20,21,26]$. Exceptions are Witte et al. [28] and Balini et al. [6], who designed continuous-time controllers which then are approximately discretized. However, Witte et al. [28] use a very high sampling frequency of $40 \mathrm{kHz}$ to reject a harmonic disturbance with a frequency up to $48 \mathrm{~Hz}$ (in fact, the authors state that they chose "the smallest [sampling time] available by the hardware") and Balini et al. [6] use a maximal sampling frequency of $50 \mathrm{kHz}$. It seems more natural to directly carry out the design in discrete time to avoid discretization issues.

\section{State-augmented observer-based state-feedback control}

Usually, disturbances act somewhere on the plant under consideration. The control objective is then to reject this disturbance. For the design methods considered in this paper, it is assumed that the disturbance acts on the plant input. For linear systems, a disturbance acting somewhere in the plant can be represented as a disturbance acting on the plant input under very mild assumptions. Therefore, assuming an input disturbance does not mean that the true disturbance has to act on the plant input (in fact, it usually does not). The design method used in this chapter is based on the internal model principle [14]. Applying this principle results in controllers with high gain in the frequency ranges where disturbances are assumed. These disturbances are then rejected no matter where they act upon the plant. 


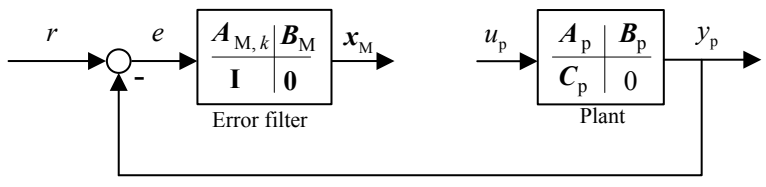

Figure 1. Interconnection of plant model and error filter

A discrete-time model for the plant with a disturbance $y_{\mathrm{d}, k}$ acting on the input is given by

$$
\begin{aligned}
x_{\mathrm{p}, k+1} & =A_{\mathrm{p}} x_{\mathrm{p}, k}+\boldsymbol{B}_{\mathrm{p}}\left(u_{\mathrm{p}, k}+y_{\mathrm{d}, k}\right), \\
y_{\mathrm{p}, k} & =C_{\mathrm{p}} x_{\mathrm{p}, k} .
\end{aligned}
$$

In the approaches presented in the following, the disturbance rejection will be achieved with observer-based state-feedback controllers. The design methods presented here use state augmentation to add certain desired dynamics to the controller. In the time-invariant case, such a state augmentation can be used to prescribe controller poles. It can easily be verified that controller poles show up as zeros in the closed-loop disturbance transfer functions. Therefore, controller poles can be chosen to correspond to disturbances that are to be suppressed.

This argumentation, of course, only holds in the time-invariant case for which transfer functions and poles are defined (and only for controller poles that are not cancelled by plant zeros). If a time-varying internal model is used, it is not easy to interpret what happens when the internal model changes. Conceptually, the argument that the controller has high (infinite) gain at the disturbance frequencies that are to be rejected should still hold. It is confirmed by experiments (see Sec. 6) that even for fairly fast changes of the disturbance frequencies, excellent disturbance rejection is achieved.

\subsection{State augmentation through an error filter}

In this approach, disturbance rejection is achieved by including additional dynamics in a state-feedback controller through error filtering. A general error filter in state-space representation is given by

$$
x_{\mathrm{M}, k+1}=\boldsymbol{A}_{\mathrm{M}} \boldsymbol{x}_{\mathrm{M}, k}+\boldsymbol{B}_{\mathrm{M}}\left(r_{k}-y_{\mathrm{p}, k}\right)
$$

and interconnected with the plant model (without disturbance input)

$$
\begin{aligned}
x_{\mathrm{p}, k+1} & =A_{\mathrm{p}} x_{\mathrm{p}, k}+B_{\mathrm{p}} u_{\mathrm{p}, k}, \\
y_{\mathrm{p}, k} & =C_{\mathrm{p}} x_{\mathrm{p}, k} .
\end{aligned}
$$

as shown in Fig. 1. Usually, the controller error $e=r-y_{\mathrm{p}}$ is used as input for the error filter. In the application considered here, however, no set-point $r$ will be given and the error filter basically acts as an output filter with input $-y_{\mathrm{p}}$. Nonetheless it will be referred to as error filter in the following.

The dynamics of the error filter can be used to include additional desired dynamics in a state-feedback controller for the overall system given by the plant and the filter. The additional 
dynamics can be chosen such that they describe the disturbances that the controller should reject, which corresponds to the internal model principle [14]. The additional dynamics can therefore be referred to as the "internal (disturbance) model." If, as in the control problem considered in this chapter, the disturbance characteristics change over time, a time-varying internal model

can be used.

$$
x_{\mathrm{M}, k+1}=A_{\mathrm{M}, k} x_{\mathrm{M}, k}-\boldsymbol{B}_{\mathrm{M}} y_{\mathrm{p}, k}
$$

In order to design a state-feedback controller for the overall system, first the overall system model is formed by combining the plant model (3) and the error filter dynamics (4) through introduction of an augmented state vector. This yields

$$
\begin{aligned}
{\left[\begin{array}{c}
x_{\mathrm{M}, k+1} \\
x_{\mathrm{p}, k+1}
\end{array}\right] } & =\left[\begin{array}{cc}
A_{\mathrm{M}, k} & -\boldsymbol{B}_{\mathrm{M}} C_{\mathrm{p}} \\
\mathbf{0} & A_{\mathrm{p}}
\end{array}\right]\left[\begin{array}{c}
x_{\mathrm{M}, k} \\
x_{\mathrm{p}, k}
\end{array}\right]+\left[\begin{array}{c}
\mathbf{0} \\
\boldsymbol{B}_{\mathrm{p}}
\end{array}\right] u_{\mathrm{p}, k} \\
y_{\mathrm{p}, k} & =\left[\begin{array}{ll}
\mathbf{0} & C_{\mathrm{p}}
\end{array}\right]\left[\begin{array}{c}
x_{\mathrm{M}, k} \\
x_{\mathrm{p}, k}
\end{array}\right] .
\end{aligned}
$$

Then, a state-feedback gain $K_{k}$ for the overall system (5) can be designed. Due to the time-varying dynamics of the error filter, the state-feedback gain may be time-varying.

Usually the plant states are not available for feedback. Therefore, an identity observer

$$
\widehat{x}_{\mathrm{p}, k+1}=\left(A_{\mathrm{p}}-L_{\mathrm{p}, k} C_{\mathrm{p}}\right) \widehat{x}_{\mathrm{p}, k}+\boldsymbol{B}_{\mathrm{p}} u_{\mathrm{p}, k}+L_{\mathrm{p}, k} y_{\mathrm{p}, k}
$$

is used to obtain an estimate $\widehat{x}_{\mathrm{p}}$ of the plant states $x_{\mathrm{p}}$. The estimate is then used for feedback instead of the unknown plant states.

For an overall state-feedback gain

$$
\boldsymbol{K}_{k}=\left[\begin{array}{ll}
\boldsymbol{K}_{\mathrm{M}, k} & \boldsymbol{K}_{\mathrm{p}, k}
\end{array}\right],
$$

where $K_{\mathrm{M}, k}$ and $K_{\mathrm{p}, k}$ denote the parts which feed back the error filter states $x_{\mathrm{M}}$ and the plant states $x_{\mathrm{p}}$ (or in this case, the estimated plant states $\widehat{x}_{\mathrm{p}}$ ), respectively, the state-feedback law is given by

$$
u_{\mathrm{p}, k}=-\boldsymbol{K}_{\mathrm{M}, k} \boldsymbol{x}_{\mathrm{M}, k}-\boldsymbol{K}_{\mathrm{p}, k} \widehat{\boldsymbol{x}}_{\mathrm{p}, k} \text {. }
$$

The structure of the overall closed-loop system is shown in Fig. 2. The plant is affected by a disturbance $y_{d}$ at the plant input. The plant output $y_{p}$ is used as input for the error filter (with a sign reversal), which outputs are its states $x_{\mathrm{M}}$, and for the identity observer for the plant. The latter gives an estimate $\widehat{x}_{\mathrm{p}}$ of the plant states computed from $y_{\mathrm{p}}$ and $u_{\mathrm{p}}$. The states are then combined and fed back with the overall state-feedback gain consisting of $K_{\mathrm{M}, k}$ and $K_{\mathrm{p}, k}$.

For stability analysis of the closed-loop system it is convenient to separate the state-space representation of the overall closed-loop dynamics including plant, observer, error filter, state-feedback and disturbance input in several parts as derived in the following. First, the controlled plant under disturbance at the input can be derived by combining (1), (6) and (8) to

$$
x_{\mathrm{p}, k+1}=\left(\boldsymbol{A}_{\mathrm{p}}-\boldsymbol{B}_{\mathrm{p}} \boldsymbol{K}_{\mathrm{p}, k}\right) \boldsymbol{x}_{\mathrm{p}, k}-\boldsymbol{B}_{\mathrm{p}} \boldsymbol{K}_{\mathrm{M}, k} \boldsymbol{x}_{\mathrm{M}, k}-\boldsymbol{B}_{\mathrm{p}} \boldsymbol{K}_{\mathrm{p}, k}\left(\widehat{\boldsymbol{x}}_{\mathrm{p}, k}-\boldsymbol{x}_{\mathrm{p}, k}\right)+\boldsymbol{B}_{\mathrm{p}} y_{\mathrm{d}, k} .
$$

It is driven by the error filter states $x_{\mathrm{M}}$, the observer error $\widehat{x}_{\mathrm{p}}-x_{\mathrm{p}}$ and the real unknown disturbance $y_{\mathrm{d}}$. 


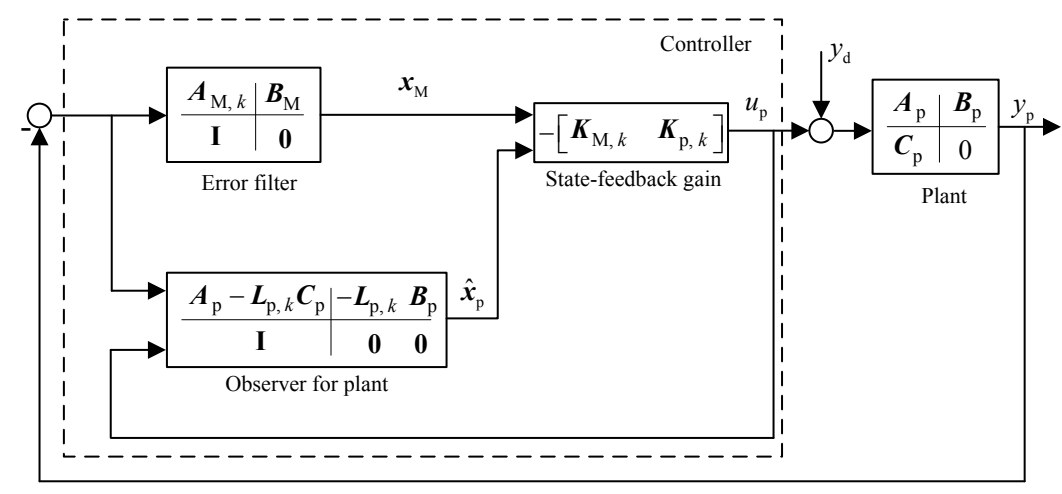

Figure 2. Structure of the resulting controller based on error filtering

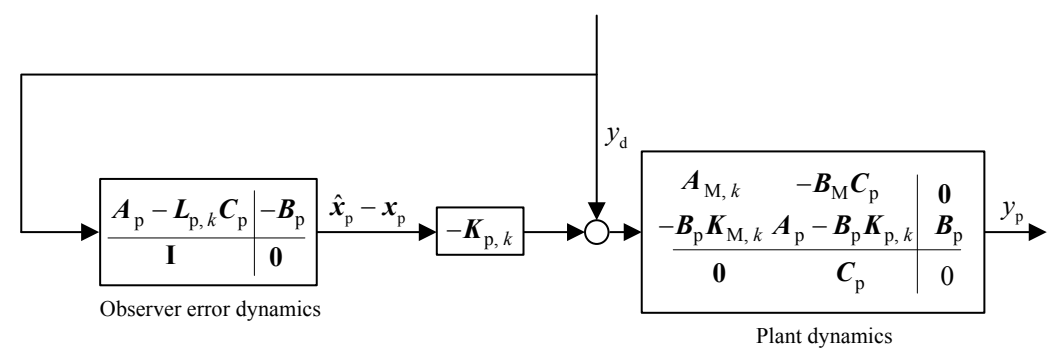

Figure 3. Dynamics of the overall closed-loop system for stability analysis

The dynamics of the identity observer for the plant states are described by (6). Since the observer does not include a model of the disturbance, the dynamics of the observer error, from now on denoted by $\widetilde{x}_{\mathrm{p}}$, are given as

$$
\widetilde{x}_{\mathrm{p}, k+1}=\left(A_{\mathrm{p}}-L_{\mathrm{p}, k} C_{\mathrm{p}}\right) \widetilde{x}_{\mathrm{p}, k}-\boldsymbol{B}_{\mathrm{p}} y_{\mathrm{d}, k}
$$

which means that the observer is driven by the unknown disturbance $y_{d}$. With the error filter dynamics given by (4), the overall system can be described as

$$
\left[\begin{array}{c}
\widetilde{\boldsymbol{x}}_{\mathrm{p}, k+1} \\
\boldsymbol{x}_{\mathrm{M}, k+1} \\
\boldsymbol{x}_{\mathrm{p}, k+1}
\end{array}\right]=\left[\begin{array}{ccc}
A_{\mathrm{p}}-\boldsymbol{L}_{\mathrm{p}, k} \boldsymbol{C}_{\mathrm{p}} & \mathbf{0} & \mathbf{0} \\
\mathbf{0} & A_{\mathrm{M}, k} & -\boldsymbol{B}_{\mathrm{M}} \boldsymbol{C}_{\mathrm{p}} \\
-\boldsymbol{B}_{\mathrm{p}} \boldsymbol{K}_{\mathrm{p}, k} & -\boldsymbol{B}_{\mathrm{p}} \boldsymbol{K}_{\mathrm{M}, k} & \boldsymbol{A}_{\mathrm{p}}-\boldsymbol{B}_{\mathrm{p}} \boldsymbol{K}_{\mathrm{p}, k}
\end{array}\right]\left[\begin{array}{c}
\widetilde{\boldsymbol{x}}_{\mathrm{p}, k} \\
\boldsymbol{x}_{\mathrm{M}, k} \\
\boldsymbol{x}_{\mathrm{p}, k}
\end{array}\right]+\left[\begin{array}{c}
-\boldsymbol{B}_{\mathrm{p}} \\
\mathbf{0} \\
\boldsymbol{B}_{\mathrm{p}}
\end{array}\right] y_{\mathrm{d}, k} .
$$

The overall system behavior is shown in Fig. 3. From this representation it can be seen that the time-varying dynamics of the augmented plant under state feedback are driven by the time-varying dynamics of the observer error for the plant states. The augmented plant under state feedback and the dynamics of the observer error for the plant states can therefore be interpreted as two distinct systems connected in series. From this it follows that choosing an observer gain $L_{\mathrm{p}, k}$ such that $A_{\mathrm{p}}-L_{\mathrm{p}, k} C_{\mathrm{p}}$ is stable and a state-feedback gain such that the time-varying dynamics of the augmented plant under state feedback are stable guarantees overall stability of the closed-loop system. 


\subsection{State augmentation through a disturbance model}

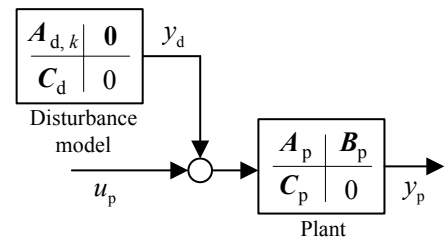

Figure 4. System model for the disturbance observer approach

In this approach, the disturbance $y_{\mathrm{d}}$ acting on the plant input $u_{\mathrm{p}}$ is explicitly modeled as the output of an unforced linear time-varying system

$$
\begin{aligned}
x_{\mathrm{d}, k+1} & =A_{\mathrm{d}, k} x_{\mathrm{d}, k} \\
y_{\mathrm{d}, k} & =C_{\mathrm{d}} x_{\mathrm{d}, k}
\end{aligned}
$$

as shown in Fig. 4. An overall linear time-varying system model is then given by the combination of the plant model (1), which is affected by the disturbance $y_{\mathrm{d}, k}$ at the plant input, and disturbance through

$$
\begin{aligned}
{\left[\begin{array}{l}
x_{\mathrm{d}, k+1} \\
x_{\mathrm{p}, k+1}
\end{array}\right] } & =\left[\begin{array}{cc}
\boldsymbol{A}_{\mathrm{d}, k} & \mathbf{0} \\
\boldsymbol{B}_{\mathrm{p}} C_{\mathrm{d}} & \boldsymbol{A}_{\mathrm{p}}
\end{array}\right]\left[\begin{array}{l}
\boldsymbol{x}_{\mathrm{d}, k} \\
\boldsymbol{x}_{\mathrm{p}, k}
\end{array}\right]+\left[\begin{array}{c}
\mathbf{0} \\
\boldsymbol{B}_{\mathrm{p}}
\end{array}\right] u_{\mathrm{p}, k}, \\
y_{\mathrm{p}, k} & =\left[\begin{array}{ll}
\mathbf{0} & C_{\mathrm{p}}
\end{array}\right]\left[\begin{array}{l}
x_{\mathrm{d}, k} \\
x_{\mathrm{p}, k}
\end{array}\right] .
\end{aligned}
$$

With

$$
x_{k}=\left[\begin{array}{c}
x_{\mathrm{d}, k} \\
x_{\mathrm{p}, k}
\end{array}\right], A_{k}=\left[\begin{array}{cc}
A_{\mathrm{d}, k} & \mathbf{0} \\
\boldsymbol{B}_{\mathrm{p}} C_{\mathrm{d}} & A_{\mathrm{p}}
\end{array}\right], \boldsymbol{B}=\left[\begin{array}{c}
\mathbf{0} \\
\boldsymbol{B}_{\mathrm{p}}
\end{array}\right], C=\left[\begin{array}{ll}
\mathbf{0} & C_{\mathrm{p}}
\end{array}\right],
$$

the overall system can be written as

$$
\begin{aligned}
& \boldsymbol{x}_{k+1}=\boldsymbol{A}_{k} \boldsymbol{x}_{k}+\boldsymbol{B} u_{\mathrm{p}, k}, \\
& y_{\mathrm{p}, k}=\boldsymbol{C} \boldsymbol{x}_{k} .
\end{aligned}
$$

Estimates for the states of the disturbance and the plant model can then be obtained by application of a linear time-varying identity observer with time-varying observer gain $\boldsymbol{L}_{k}$

$$
\widehat{\boldsymbol{x}}_{k+1}=\left(\boldsymbol{A}_{k}-\boldsymbol{L}_{k} \boldsymbol{C}\right) \widehat{\boldsymbol{x}}_{k}+\boldsymbol{B} u_{\mathrm{p}, k}+\boldsymbol{L}_{k} y_{\mathrm{p}, k} .
$$

A state-feedback control for the overall system is given by

$$
u_{\mathrm{p}, k}=-\boldsymbol{K}_{k} \widehat{\boldsymbol{x}}_{k} .
$$

This leads to the typical structure of an observer-based state-feedback controller, but due to the time-varying disturbance model, the observer gain as well as state-feedback gain might be time-varying. Fig. 5 shows the general structure. The idea behind the disturbance observer is to use the estimate of the disturbance states (with a sign reversal) as control input. This should 


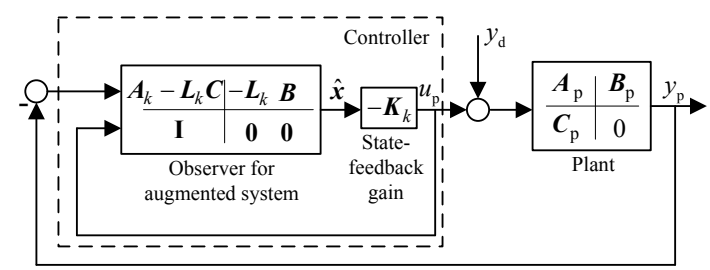

Figure 5. Observer-based state-feedback control in the disturbance observer approach

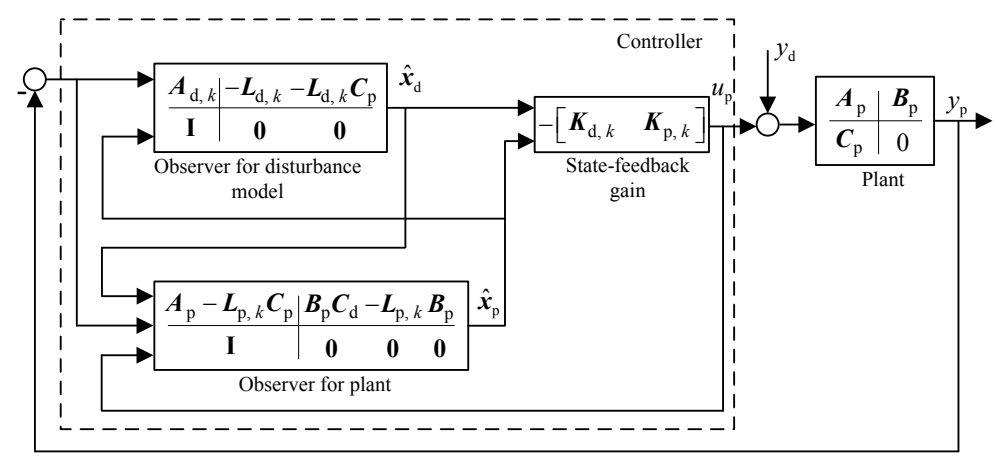

Figure 6. Detailed illustration of observer structure

lead to perfect disturbance cancellation if the disturbance model is chosen appropriately. For a more detailed analysis of this aspect, in the following observer gains and state-feedback gains are seperated into a part that feeds back the disturbance states via $L_{\mathrm{d}, k}$ and $K_{\mathrm{d}, k}$ and a part that feeds back the plant states or their estimates via $L_{\mathrm{p}, k}$ and $K_{\mathrm{p}, k}$. The estimated disturbance states can be described by

$$
\widehat{x}_{\mathrm{d}, k+1}=A_{\mathrm{d}, k} \widehat{x}_{\mathrm{d}, k}-L_{\mathrm{d}, k} C_{\mathrm{p}} \widehat{x}_{\mathrm{p}, k}+L_{\mathrm{d}, k} y_{\mathrm{p}, k},
$$

and the estimated plant states are given through

$$
\widehat{x}_{\mathrm{p}, k+1}=\left(A_{\mathrm{p}}-\boldsymbol{L}_{\mathrm{p}, k} \boldsymbol{C}_{\mathrm{p}}\right) \widehat{\boldsymbol{x}}_{\mathrm{p}, k}+\boldsymbol{B}_{\mathrm{p}} \boldsymbol{C}_{\mathrm{d}} \widehat{\boldsymbol{x}}_{\mathrm{d}, k}+\boldsymbol{L}_{\mathrm{p}, k} y_{\mathrm{p}, k}+\boldsymbol{B}_{\mathrm{p}} u_{\mathrm{p}, k}
$$

where

$$
\boldsymbol{L}_{k}=\left[\begin{array}{ll}
\boldsymbol{L}_{\mathrm{d}, k} & \boldsymbol{L}_{\mathrm{p}, k}
\end{array}\right] .
$$

In Fig. 6, this more detailed representation is illustrated.

For stability analysis of the overall system including overall observer dynamics and state feedback, the dynamics of the observer error for the plant states

$$
\widehat{x}_{\mathrm{p}, k+1}-x_{\mathrm{p}, k+1}=\left(A_{\mathrm{p}}-L_{\mathrm{p}, k} C_{\mathrm{p}}\right)\left(\widehat{x}_{\mathrm{p}, k}-x_{\mathrm{p}, k}\right)+\boldsymbol{B}_{\mathrm{p}} C_{\mathrm{d}} \widehat{x}_{\mathrm{d}, k}-\boldsymbol{B}_{\mathrm{p}} y_{\mathrm{d}, k}
$$

are considered.

The dynamics of the states of the plant under state feedback are given by

$$
x_{\mathrm{p}, k+1}=\left(A_{\mathrm{p}}-\boldsymbol{B}_{\mathrm{p}} \boldsymbol{K}_{\mathrm{p}, k}\right) \boldsymbol{x}_{\mathrm{p}, k}-\boldsymbol{B}_{\mathrm{p}} \boldsymbol{K}_{\mathrm{d}, k} \widehat{\boldsymbol{x}}_{\mathrm{d}, k}+\boldsymbol{B}_{\mathrm{p}} \boldsymbol{K}_{\mathrm{p}, k}\left(\widehat{\boldsymbol{x}}_{\mathrm{p}, k}-\boldsymbol{x}_{\mathrm{p}, k}\right)+\boldsymbol{B}_{\mathrm{p}} y_{\mathrm{d}, k} .
$$




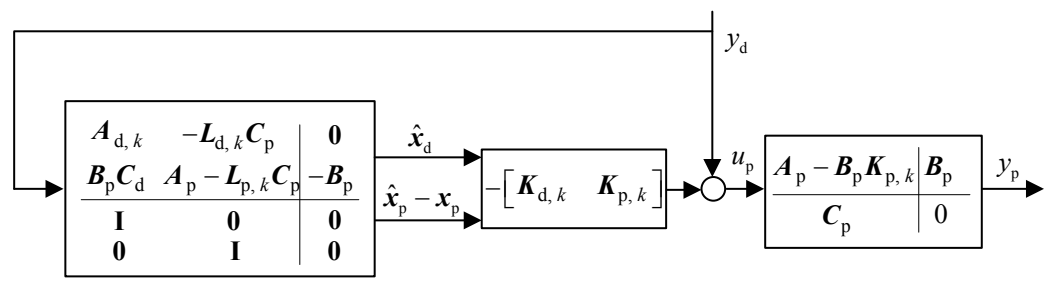

Figure 7. Dynamics of the overall closed-loop system for stability analysis

This yields the overall closed-loop system dynamics given by

$$
\left[\begin{array}{c}
\widehat{x}_{\mathrm{d}, k+1} \\
\widetilde{\boldsymbol{x}}_{\mathrm{p}, k+1} \\
\boldsymbol{x}_{\mathrm{p}, k+1}
\end{array}\right]=\left[\begin{array}{ccc}
\boldsymbol{A}_{\mathrm{d}, k} & -\boldsymbol{L}_{\mathrm{d}, k} \boldsymbol{C}_{\mathrm{p}} & \mathbf{0} \\
\boldsymbol{B}_{\mathrm{p}} \boldsymbol{C}_{\mathrm{d}} & A_{\mathrm{p}}-\boldsymbol{L}_{\mathrm{p}, k} \boldsymbol{C}_{\mathrm{p}} & \mathbf{0} \\
-\boldsymbol{B}_{\mathrm{p}} \boldsymbol{K}_{\mathrm{d}, k} & \boldsymbol{B}_{\mathrm{p}} \boldsymbol{K}_{\mathrm{p}, k} & \boldsymbol{A}_{\mathrm{p}}-\boldsymbol{B}_{\mathrm{p}} \boldsymbol{K}_{\mathrm{p}, k}
\end{array}\right]\left[\begin{array}{c}
\widehat{\boldsymbol{x}}_{\mathrm{d}, k} \\
\widetilde{\boldsymbol{x}}_{\mathrm{p}, k} \\
\boldsymbol{x}_{\mathrm{p}, k}
\end{array}\right]+\left[\begin{array}{c}
\mathbf{0} \\
-\boldsymbol{B}_{\mathrm{p}} \\
\boldsymbol{B}_{\mathrm{p}}
\end{array}\right] y_{\mathrm{d}, k} .
$$

Fig. 7 illustrates the structure of the overall system dynamics. This representation shows, similiar to the error filtering approach, that the dynamics of the plant under state feedback are driven by the time-varying dynamics of the observer for the augmented system, where in this case the outputs are given by the estimate of the disturbance states and the observer error for the plant states. The plant under state feedback and the observer of the augmented system can therefore be considered as two distinct systems in series connection. From this it follows that as long as a stabilizing state-feedback gain $\boldsymbol{K}_{\mathrm{p}, k}$ for the linear time-invariant plant and a stabilizing observer gain for the augmented system model is chosen, the overall closed-loop system is stable. It is also evident that the choice of the disturbance model does not influence the overall system stability, as long as the disturbance model itself is stable. Also, the choice of $\boldsymbol{K}_{\mathrm{d}, k}$ has no effect on the overall system stability (as long as $\boldsymbol{K}_{\mathrm{d}, k} \widehat{\boldsymbol{x}}_{\mathrm{d}, k}$ remains bounded). Therefore, the overall state-feedback gain $K$ does not have to be time-varying since only the linear time-invariant plant must be stabilized by $K_{\mathrm{p}}$ and the choice of $\boldsymbol{K}_{\mathrm{d}}$ is not important for system stability. Furthermore, it can be seen that, assuming a perfect disturbance model, complete disturbance cancellation will be achieved after some transient if $K_{\mathrm{d}}=C_{\mathrm{d}}$.

\section{Gain-scheduled state-feedback and observer design for pLPV systems}

In this section, design methods for time-varying state-feedback and observer gains for pLPV systems based on quadratic stability theory are reviewed.

An LPV system is a linear system described by

$$
\begin{aligned}
\boldsymbol{x}_{k+1} & =\boldsymbol{A}(\boldsymbol{\theta}) \boldsymbol{x}_{k}+\boldsymbol{B}(\boldsymbol{\theta}) \boldsymbol{u}_{k} \\
\boldsymbol{y}_{k} & =\boldsymbol{C}(\boldsymbol{\theta}) \boldsymbol{x}_{k}+\boldsymbol{D}(\boldsymbol{\theta}) \boldsymbol{u}_{k}
\end{aligned}
$$

with $x_{k} \in \mathbb{R}^{n}$, where the state-space matrices depend on a possibly time-varying parameter vector

$$
\boldsymbol{\theta}=\left[\theta_{1} \theta_{2} \cdots \theta_{N}\right]^{\mathrm{T}} \in \boldsymbol{\Theta} \subset \mathbb{R}^{N} .
$$

In the following, only unforced systems of the form

$$
x_{k+1}=A(\theta) x_{k}
$$


are considered. In general, an LPV system is called a pLPV system, if the matrices depend affinely on the varying parameter. For the unforced system given in (26), this means that it is a pLPV system, if the system matrix $A(\cdot)$ depends affinely on $\theta$, that is

$$
\boldsymbol{A}(\boldsymbol{\theta})=\mathcal{A}_{0}+\theta_{1} \mathcal{A}_{1}+\theta_{2} \mathcal{A}_{2}+\cdots+\theta_{N} \mathcal{A}_{N}
$$

where $\mathcal{A}_{i} \in \mathbb{R}^{n \times n}$ for all $i=0,1, \ldots, N$ are constant matrices and $\boldsymbol{\Theta}$ is a convex polytope in $\mathbb{R}^{N}$ with a finite set of vertices $V=\left\{\boldsymbol{v}_{1}, \boldsymbol{v}_{2}, \ldots, \boldsymbol{v}_{M}\right\} \subset \mathbb{R}^{N}$. A point $\boldsymbol{\theta} \in \boldsymbol{\Theta}$ can be written as a convex combination of vertices, which means there exists a coordinate vector $\lambda=\left[\begin{array}{llll}\lambda_{1} & \lambda_{2} & \ldots & \lambda_{M}\end{array}\right]^{\mathrm{T}} \in \mathbb{R}^{M}$ such that $\boldsymbol{\theta}$ can be written as

$$
\boldsymbol{\theta}=\sum_{j=1}^{M} \lambda_{j} \boldsymbol{v}_{j}, \quad \lambda_{j} \geq 0 \quad \forall j=1, \ldots, M, \quad \sum_{j=1}^{M} \lambda_{j}=1 .
$$

Defining $A_{\mathrm{v}, j}=\boldsymbol{A}\left(\boldsymbol{v}_{j}\right)$ for $j=1, \ldots, M, \boldsymbol{A}(\boldsymbol{\theta})$ can be represented as

$$
\boldsymbol{A}(\boldsymbol{\theta})=\boldsymbol{A}(\boldsymbol{\lambda})=\lambda_{1} A_{\mathrm{v}, 1}+\lambda_{2} A_{\mathrm{v}, 2}+\cdots+\lambda_{M} A_{\mathrm{v}, M}
$$

The matrices $A_{\mathrm{v}, j}$ can be considered as system matrices of linear time-invariant vertex systems

$$
x_{k+1}=A_{\mathrm{v}, j} x_{k}, j=1, \ldots, M,
$$

and the current system (26) is a convex combination of the vertex systems in (30).

The pLPV system (26) is called quadratically stable in $\boldsymbol{\Theta}$ if and only if there exists a positive definite matrix $\boldsymbol{P}$ such that for all $\boldsymbol{\theta} \in \boldsymbol{\Theta}$

$$
A^{\mathrm{T}}(\boldsymbol{\theta}) \boldsymbol{P A}(\boldsymbol{\theta})-\boldsymbol{P}<0,
$$

or equivalently via the Schur complement [18]

$$
\left[\begin{array}{cc}
\boldsymbol{P} & \boldsymbol{P A}(\boldsymbol{\theta}) \\
\boldsymbol{A}^{\mathrm{T}}(\boldsymbol{\theta}) \boldsymbol{P} & \boldsymbol{P}
\end{array}\right]>0
$$

where " $>0$ " and " $<0$ " indicate positive and negative definiteness, respectively.

Since (32) has to hold for every $\boldsymbol{\theta}$, this constitutes an infinite set of linear matrix inequalities (LMIs), which is computationally intractable. By the following result, it is possible to use a finite set of inequalities. It holds that a pLPV system in the form of (26) is quadratically stable for every $\boldsymbol{\theta} \in \boldsymbol{\Theta}$ if and only if there exists a symmetric positive definite matrix $\boldsymbol{P}$ such that

$$
A_{\mathrm{v}, j}^{\mathrm{T}} \boldsymbol{P} A_{\mathrm{v}, j}-P<0,
$$

or equivalently

$$
\left[\begin{array}{cc}
\boldsymbol{P} & \boldsymbol{P} A_{\mathrm{v}, j} \\
\boldsymbol{A}_{\mathrm{v}, j}^{\mathrm{T}} \boldsymbol{P} & \boldsymbol{P}
\end{array}\right]>0,
$$

for all $j=1, \ldots, M$. This result is based on one single quadratic Lyapunov function that assures stability for the whole parameter space $\boldsymbol{\Theta}$. Therefore, $\boldsymbol{\theta}$ is allowed to change arbitrarily fast with time and $\boldsymbol{\theta}$ and $\lambda$ can explicitly be assumed time-varying and denoted as $\boldsymbol{\theta}_{k}$ and $\lambda_{k}$, respectively. A proof is given in Amato [1]. 
These results can be applied to the design of time-varying state-feedback and observer gains, as reviewed in the following sections.

\subsection{State-feedback design for pLPV systems based on quadratic stability}

A system of the form

$$
\boldsymbol{x}_{k+1}=\boldsymbol{A}\left(\boldsymbol{\theta}_{k}\right) \boldsymbol{x}_{k}+\boldsymbol{B} \boldsymbol{u}_{k}
$$

is considered and the objective is to find a state-feedback gain $K\left(\boldsymbol{\theta}_{k}\right)$ that quadratically stabilizes the closed-loop system

$$
\boldsymbol{x}_{k+1}=\left(\boldsymbol{A}\left(\boldsymbol{\theta}_{k}\right)-\boldsymbol{B} \boldsymbol{K}\left(\boldsymbol{\theta}_{k}\right)\right) \boldsymbol{x}_{k} .
$$

From the above result it follows that it suffices to find a single symmetric positive definite matrix $\boldsymbol{P}$ and a finite set of matrices $\boldsymbol{K}_{\mathrm{v}, j}$ such that

$$
\left[\begin{array}{cc}
\boldsymbol{P} & \boldsymbol{P}\left(\boldsymbol{A}_{\mathrm{v}, j}-\boldsymbol{B} \boldsymbol{K}_{\mathrm{v}, j}\right) \\
\left(\boldsymbol{A}_{\mathrm{v}, j}-\boldsymbol{B} \boldsymbol{K}_{\mathrm{v}, j}\right)^{\mathrm{T}} \boldsymbol{P} & \boldsymbol{P}
\end{array}\right]>0, j=1, \ldots, M .
$$

Then, $\boldsymbol{K}\left(\boldsymbol{\theta}_{k}\right)$ has to be chosen as

$$
\boldsymbol{K}\left(\boldsymbol{\theta}_{k}\right)=\sum_{j=1}^{M} \lambda_{j, k} \boldsymbol{K}_{\mathrm{v}, j}=\boldsymbol{K}\left(\boldsymbol{\lambda}_{k}\right)
$$

because then it holds that

$$
\boldsymbol{A}\left(\boldsymbol{\theta}_{k}\right)-\boldsymbol{B} \boldsymbol{K}\left(\boldsymbol{\theta}_{k}\right)=\sum_{j=1}^{M} \lambda_{j, k} \boldsymbol{A}_{\mathrm{v}, j}-\boldsymbol{B} \sum_{j=1}^{M} \lambda_{j, k} \boldsymbol{K}_{\mathrm{v}, j}=\sum_{j=1}^{M} \lambda_{j, k}\left(\boldsymbol{A}_{\mathrm{v}, j}-\boldsymbol{B} \boldsymbol{K}_{\mathrm{v}, j}\right),
$$

and therefore quadratic stability of (36) is implied due to the results presented above.

\subsection{Computation of state-feedback gains for the vertex systems}

In this section, sufficient conditions that guarantee closed-loop stability and a certain $\mathrm{H}_{2}$ performance level for the controlled vertex systems that can be used to calculate state-feedback gains for the vertex systems are derived. To use an $H_{2}$ performance level, an additional performance input is introduced that enters the state update equation for each vertex system of the pLPV system in (35). This input can be interpreted as process noise. For every $j=1, \ldots, M$, this yields

$$
x_{k+1}=A_{\mathrm{v}, j} x_{k}+B u_{k}+w_{k} .
$$

As a performance output, the artificial signal

$$
z_{k}=\left[\begin{array}{l}
Q^{\frac{1}{2}} \boldsymbol{x}_{k} \\
\boldsymbol{R}^{\frac{1}{2}} \boldsymbol{u}_{k}
\end{array}\right]
$$

is defined that weighs the states and the control signal with matrices $Q^{\frac{1}{2}}$ and $\boldsymbol{R}^{\frac{1}{2}}$, respectively. The objective then is to find state-feedback gains $K_{\mathrm{v}, j}$ that stabilize the system and minimize the $\mathrm{H}_{2}$ norm of the transfer path from $\boldsymbol{w}_{k}$ to $z_{k}$, when the control signal $\boldsymbol{u}_{k}$ in (40) and (41) is 
chosen as

$$
\boldsymbol{u}_{k}=-\boldsymbol{K}_{\mathrm{v}, j} \boldsymbol{x}_{k} .
$$

This objective can be stated as the minimization of the $H_{2}$ norm of the system

$$
z=G w
$$

which has the state-space representation

$$
\begin{aligned}
x_{k+1} & =\widetilde{A} x_{k}+\widetilde{B} w_{k} \\
z_{k} & =\widetilde{C} x_{k}
\end{aligned}
$$

with

$$
\widetilde{A}=A_{\mathrm{v}, j}-B K_{\mathrm{v}, j}, \quad \widetilde{B}=\mathbf{I}, \quad \widetilde{\boldsymbol{C}}=\left[\begin{array}{c}
Q^{\frac{1}{2}} \\
-\boldsymbol{R}^{\frac{1}{2}} K_{\mathrm{v}, j}
\end{array}\right] .
$$

If the system is stable, the $\mathrm{H}_{2}$ norm of this discrete-time linear time-invariant system is given by

$$
\|G\|_{2}^{2}=\operatorname{trace}\left(\widetilde{\boldsymbol{C}} \boldsymbol{W}_{\mathrm{c}} \widetilde{\boldsymbol{C}}^{\mathrm{T}}\right),
$$

where the controllability gramian $\boldsymbol{W}_{\mathrm{c}}$ satisfies the discrete-time Lyapunov equation

$$
\widetilde{A} W_{\mathrm{c}} \widetilde{A}^{\mathrm{T}}-W_{\mathrm{c}}+\widetilde{B} \widetilde{B}^{\mathrm{T}}=\mathbf{0} .
$$

Therefore, if there exist symmetric positive definite matrices $\boldsymbol{P}$ and $\boldsymbol{W}$ such that

$$
\begin{aligned}
& \widetilde{A} \boldsymbol{P} \widetilde{\boldsymbol{A}}^{\mathrm{T}}-\boldsymbol{P}+\widetilde{\boldsymbol{B}} \widetilde{\boldsymbol{B}}^{\mathrm{T}}<0, \\
& \boldsymbol{W}-\widetilde{\boldsymbol{C}} \boldsymbol{P} \widetilde{\boldsymbol{C}}^{\mathrm{T}}>0, \\
& \text { trace }(\boldsymbol{W})<\gamma^{2} \text {, }
\end{aligned}
$$

then it follows that $\|G\|_{2}<\gamma$ for a $\gamma>0$. Through the Schur complement, (48) and (49) can be transformed to

Introducing

$$
\begin{gathered}
{\left[\begin{array}{cc}
\boldsymbol{P} & \boldsymbol{P} \widetilde{A}^{\mathrm{T}} \\
\widetilde{A} \boldsymbol{P} \boldsymbol{P}-\widetilde{\boldsymbol{B}} \widetilde{\boldsymbol{B}}^{\mathrm{T}}
\end{array}\right]>0,} \\
{\left[\begin{array}{cc}
\boldsymbol{W} & \widetilde{\boldsymbol{C}} \boldsymbol{P} \\
\boldsymbol{P} \widetilde{\boldsymbol{C}}^{\mathrm{T}} & \boldsymbol{P}
\end{array}\right]>0 .}
\end{gathered}
$$

$$
\widetilde{\boldsymbol{Q}}=\left[\begin{array}{c}
\boldsymbol{Q}^{\frac{1}{2}} \\
\mathbf{0}
\end{array}\right], \quad \widetilde{\boldsymbol{R}}=\left[\begin{array}{c}
\mathbf{0} \\
\boldsymbol{R}^{\frac{1}{2}}
\end{array}\right], \quad \boldsymbol{Y}_{\mathrm{v}, j}=\boldsymbol{K}_{\mathrm{v}, j} \boldsymbol{P},
$$

it follows that if solutions for the matrix variables $\boldsymbol{P}, \boldsymbol{W}$ and $Y_{\mathrm{v}, j}, j=1, \ldots, M$, can be found that satisfy the $2 M+1$ LMIs

$$
\left[\begin{array}{cc}
\boldsymbol{P} & \left(\boldsymbol{A}_{\mathrm{v}, j} \boldsymbol{P}-\boldsymbol{B} \boldsymbol{Y}_{\mathrm{v}, j}\right)^{\mathrm{T}} \\
\boldsymbol{A}_{\mathrm{v}, j} \boldsymbol{P}-\boldsymbol{B} \boldsymbol{Y}_{\mathrm{v}, j} & \boldsymbol{P}-\mathbf{I}
\end{array}\right]>0, j=1, \ldots, M,
$$




$$
\left[\begin{array}{cc}
\boldsymbol{W} & \widetilde{Q} \boldsymbol{P}-\widetilde{\boldsymbol{R}} \boldsymbol{Y}_{\mathrm{v}, j} \\
\left(\widetilde{\boldsymbol{Q}} \boldsymbol{P}-\widetilde{\boldsymbol{R}} \boldsymbol{Y}_{\mathrm{v}, j}\right)^{\mathrm{T}} & \boldsymbol{P}
\end{array}\right]>0, j=1, \ldots, M,
$$

then the system $\boldsymbol{G}$ has an $\mathrm{H}_{2}$ norm bounded by $\gamma$. From the solutions $\boldsymbol{P}$ and $\boldsymbol{Y}_{\mathrm{v}, j}$, the state-feedback gain for each vertex system can be calculated as

$$
K_{\mathrm{v}, j}=\boldsymbol{Y}_{\mathrm{v}, j} \boldsymbol{P}^{-1}
$$

Quadratic stability is then implied for each closed-loop vertex system because of (48). In order to guarantee quadratic stability for the whole parameter space, solutions for the matrix variables $\boldsymbol{P}$ and $\boldsymbol{W}$ in (54)-(56) have to be the same for all vertex systems. Therefore, if solutions are found, also the performance bound $\gamma$ is guaranteed for every fixed $\boldsymbol{\theta}$ in the parameter space $\boldsymbol{\Theta}$, since it depends only on $\boldsymbol{P}$ and $\boldsymbol{W}$.

Once state-feedback gains are found for the vertex systems, in any instant of time of a realization of the pLPV system (35) with $\boldsymbol{\theta}_{k} \in \boldsymbol{\Theta}$, a state-feedback gain can be found via interpolation according to (38). Quadratic stability of the closed-loop system (36) is guaranteed. The question of how to compute the coordinates $\lambda_{k}$ with the properties described in (28) depends on the specific polytope $\Theta$. The case of an $N$-dimensional hyper box will be considered in detail in Sec. 4.4 .

\subsection{Observer design for PLPV systems based on quadratic stability}

Although the observer design is dual to the controller design, the LMIs for observer design are shortly presented here for completeness.

For observer design, a system of the form

$$
\begin{aligned}
x_{k+1} & =A\left(\theta_{k}\right) x_{k}+B u_{k} \\
y_{k} & =C x_{k}
\end{aligned}
$$

is considered and the objective is to find an observer gain $\boldsymbol{L}\left(\boldsymbol{\theta}_{k}\right)$ that quadratically (and therefore, asymptotically) stabilizes the dynamics of the observer error

$$
\widetilde{\boldsymbol{x}}_{k+1}=\left(\boldsymbol{A}\left(\boldsymbol{\theta}_{k}\right)-\boldsymbol{L}\left(\boldsymbol{\theta}_{k}\right) \boldsymbol{C}\right) \widetilde{\boldsymbol{x}}_{k} .
$$

From the above result it follows that it suffices to find a single symmetric positive definite matrix $\boldsymbol{P}$ and a finite set of matrices $L_{\mathrm{v}, j}$ such that

$$
\left[\begin{array}{cc}
\boldsymbol{P} & \boldsymbol{P}\left(\boldsymbol{A}_{\mathrm{v}, j}-\boldsymbol{L}_{\mathrm{v}, j} \boldsymbol{C}\right) \\
\left(\boldsymbol{A}_{\mathrm{v}, j}-\boldsymbol{L}_{\mathrm{v}, j} \boldsymbol{C}\right)^{\mathrm{T}} \boldsymbol{P} & \boldsymbol{P}
\end{array}\right]>0, j=1, \ldots, M,
$$

and $\boldsymbol{L}\left(\boldsymbol{\theta}_{k}\right)$ has to be chosen as

$$
\boldsymbol{L}\left(\boldsymbol{\theta}_{k}\right)=\sum_{j=1}^{M} \lambda_{j, k} \boldsymbol{L}_{\mathrm{v}, j}=\boldsymbol{L}\left(\boldsymbol{\lambda}_{k}\right) .
$$


As in the design of the state-feedback gain in the previous section, sufficient conditions for quadratic stability of (59) and a certain $\mathrm{H}_{2}$ performance level can be derived. It is assumed that white noise of unit covariance, given by $w_{1}$ and $w_{2}$ and weighted by matrices $Q^{\frac{1}{2}}$ and $R^{\frac{1}{2}}$ respectively, affects the states and the outputs of the vertex systems of (58). This gives

$$
\begin{aligned}
\boldsymbol{x}_{k+1} & =\boldsymbol{A}\left(\boldsymbol{\theta}_{k}\right) \boldsymbol{x}_{k}+C \boldsymbol{u}_{k}+Q^{\frac{1}{2}} \boldsymbol{w}_{1, k} \\
\boldsymbol{y}_{k} & =\boldsymbol{C} \boldsymbol{x}_{k}+\boldsymbol{R}^{\frac{1}{2}} \boldsymbol{w}_{2, k} .
\end{aligned}
$$

The $\mathrm{H}_{2}$-norm of the transfer path from this noise input to the observer error is chosen as the performance measure. Defining

$$
\boldsymbol{w}_{k}=\left[\begin{array}{c}
w_{1, k} \\
w_{2, k}
\end{array}\right]
$$

this transfer path is described by

$$
\widetilde{\boldsymbol{x}}_{k+1}=\left(A_{\mathrm{v}, j}-\boldsymbol{L}_{\mathrm{v}, k} C\right) \widetilde{\boldsymbol{x}}_{k}+\left[Q^{\frac{1}{2}}-\boldsymbol{L}_{\mathrm{v}, k} \boldsymbol{R}^{\frac{1}{2}}\right] \boldsymbol{w}_{k} .
$$

Therefore the objective is to minimize the $H_{2}$-norm of the system $z=G w$ with state-space representation

$$
\begin{aligned}
\boldsymbol{x}_{k+1} & =\widetilde{\boldsymbol{A}} \boldsymbol{x}_{k}+\widetilde{\boldsymbol{B}} \boldsymbol{w}_{k} \\
z_{k} & =\widetilde{\boldsymbol{C}} \boldsymbol{x}_{k}
\end{aligned}
$$

with

$$
\widetilde{A}=A_{\mathrm{v}, j}-L_{\mathrm{v}, j} C, \quad \widetilde{B}=\left[Q^{\frac{1}{2}}-L_{\mathrm{v}, k} R^{\frac{1}{2}}\right], \quad \widetilde{C}=\mathbf{I} .
$$

If the system is stable, the $\mathrm{H}_{2}$ norm of this discrete-time linear time-invariant system is given by

$$
\|G\|_{2}^{2}=\operatorname{trace}\left(\widetilde{\boldsymbol{B}}^{\mathrm{T}} \boldsymbol{W}_{\mathrm{o}} \widetilde{\boldsymbol{B}}\right),
$$

where the observability gramian $\boldsymbol{W}_{\mathrm{o}}$ satisfies the discrete-time Lyapunov equation

$$
\widetilde{A} W_{\mathrm{o}} \widetilde{A}^{\mathrm{T}}-W_{\mathrm{O}}+\widetilde{C}^{\mathrm{T}} \widetilde{C}=\mathbf{0} .
$$

For the same arguments as in Sec. 4.1 and with the introduction of

$$
\widetilde{\boldsymbol{Q}}=\left[\begin{array}{c}
\boldsymbol{Q}^{\frac{\mathrm{T}}{2}} \\
\mathbf{0}
\end{array}\right], \quad \widetilde{\boldsymbol{R}}=\left[\begin{array}{c}
\mathbf{0} \\
\boldsymbol{R}^{\frac{\mathrm{T}}{2}}
\end{array}\right], \quad \boldsymbol{Y}_{\mathrm{v}, j}=\boldsymbol{L}_{\mathrm{v}, j}^{\mathrm{T}} \boldsymbol{P}, \quad j=1, \ldots, M,
$$

it follows that if solutions for the matrix variables $\boldsymbol{P}, \boldsymbol{W}$ and $\boldsymbol{Y}_{\mathrm{v}, j}, j=1, \ldots, M$, can be found that satisfy the $2 M+1$ LMIs

$$
\begin{gathered}
{\left[\begin{array}{cc}
\boldsymbol{P} & \boldsymbol{P} \boldsymbol{A}_{\mathrm{v}, j}-\boldsymbol{Y}_{\mathrm{v}, j}^{\mathrm{T}} \boldsymbol{C} \\
\left(\boldsymbol{P} A_{\mathrm{v}, j}-\boldsymbol{Y}_{\mathrm{v}, j}^{\mathrm{T}} \boldsymbol{C}\right)^{\mathrm{T}} & \boldsymbol{P}-\mathbf{I}
\end{array}\right]>0, j=1, \ldots, M,} \\
{\left[\begin{array}{cc}
\boldsymbol{W} & \widetilde{\boldsymbol{Q}} \boldsymbol{P}-\widetilde{\boldsymbol{R}} \boldsymbol{Y}_{\mathrm{v}, j} \\
\left(\widetilde{\boldsymbol{Q}} \boldsymbol{P}-\widetilde{\boldsymbol{R}} \boldsymbol{Y}_{\mathrm{v}, j}\right)^{\mathrm{T}} & \boldsymbol{P}
\end{array}\right]>0, j=1, \ldots, M,}
\end{gathered}
$$




$$
\operatorname{trace}(\boldsymbol{W})<\gamma^{2}
$$

then the system $\boldsymbol{G}$ has an $\mathrm{H}_{2}$ norm bounded by $\gamma$. From the solutions $\boldsymbol{P}$ and $\boldsymbol{Y}_{\mathrm{v}, j}$, the observer gain for each vertex system can be calculated as

$$
\boldsymbol{L}_{\mathrm{v}, j}=\boldsymbol{P}^{-\mathrm{T}} \boldsymbol{Y}_{\mathrm{v}, j}^{\mathrm{T}}
$$

In order to guarantee quadratic stability for the whole parameter space, solutions for the matrix variables $\boldsymbol{P}$ and $\boldsymbol{W}$ in (70)-(72) have to be the same for all vertex systems. Therefore, if solutions are found, also the performance bound $\gamma$ is guaranteed for every fixed $\boldsymbol{\theta}$ in the parameter space $\Theta$.

Once observer gains are found for the vertex systems, in any instant of time an observer gain for the realization of the pLPV system (58) with $\boldsymbol{\theta}_{k} \in \boldsymbol{\Theta}$ can be found via interpolation according to (61).

\subsection{Computation of the coordinates for interpolation}

The computation of the coordinates $\lambda_{k} \in \mathbb{R}^{M}$ required for the interpolation depends on the specific polytope $\Theta \subset \mathbb{R}^{N}$ that is used to cover the parameter space. In general, for a parameter vector $\boldsymbol{\theta}_{k} \in \boldsymbol{\Theta}$, where $\boldsymbol{\Theta}$ denotes a polytope with $M$ vertices $\boldsymbol{v}_{1}, \boldsymbol{v}_{2}, \ldots, \boldsymbol{v}_{M} \in \mathbb{R}^{N}$, the coordinate vector $\lambda_{k}$ as required for the interpolation is given by the solution to the constrained system of linear equations

$$
\left[\begin{array}{cccc}
\boldsymbol{v}_{1} & \boldsymbol{v}_{2} & \ldots & \boldsymbol{v}_{M} \\
1 & 1 & \ldots & 1
\end{array}\right] \lambda_{k}=\left[\begin{array}{c}
\boldsymbol{\theta}_{k} \\
1
\end{array}\right], 0 \leq \lambda_{j, k} \leq 1, j=1, \ldots, M
$$

Existence of the solution is guaranteed as long as $\boldsymbol{\theta}_{k} \in \boldsymbol{\Theta}$. If the polytope considered has less than or exactly $N+1$ vertices, the constrained linear equation system admits exactly one solution. If the polytope is given by more than $N+1$ vertices, the system is underdetermined and more than one solution is possible. Since the controller design is not a linear operation, different choices of coordinates might lead to different properties of the resulting controller.

In most cases, ranges of components of the parameter vector are given by closed intervals in $\mathbb{R}$ determined by upper and lower bounds, such that

$$
\theta_{i, k} \in\left[\theta_{i, \min }, \theta_{i, \max }\right], i=1, \ldots, N .
$$

If no further information on the relations between parameters are known, the polytope $\Theta$ has to be chosen as the $N$-dimensional hyper box, also referred to as the parameter box, given by

$$
\boldsymbol{\Theta}=\left[\theta_{1, \min }, \theta_{1, \max }\right] \times\left[\theta_{2, \min }, \theta_{2, \max }\right] \times \ldots \times\left[\theta_{N, \min }, \theta_{N, \max }\right] .
$$

One way to compute a set of coordinates $\lambda_{k}$ for this case is introduced by Apkarian et al. [2] and generalized and implemented for an arbitrary number of vertices in the LMI Control Toolbox for Matlab [15]. Another implementation of this approach is proposed here that is suitable for real-time implementation purposes where variables have to be pre-initialized with fixed dimensions. Daafouz et al. [9] presented a compact way of writing the calculation scheme, on which the scheme proposed here and in [17] is based. If the order of vertices is not changed, any of the mentioned approaches leads to the same coordinates. 
The $i$-th entry $v_{j, i}$ of a vertex $j$ of the parameter box $\Theta$ is either the lower bound $\theta_{i, \min }$ or the upper bound $\theta_{i, \max }$ of $\theta_{i, k}$. Now, $2 N$ vectors

$$
\boldsymbol{b}_{i_{\min }}=\left[\begin{array}{c}
b_{i_{\min }, 1} \\
\vdots \\
b_{i_{\min }, M}
\end{array}\right], \boldsymbol{b}_{i_{\max }}=\left[\begin{array}{c}
b_{i_{\max }, 1} \\
\vdots \\
b_{i_{\max }, M}
\end{array}\right]
$$

are pre-computed such that

$$
\begin{aligned}
& b_{i_{\max }, j}=\left\{\begin{array}{cl}
\frac{1}{\theta_{i, \max }-\theta_{i, \min },} & \text { if } v_{j, i}=\theta_{i, \max } \\
0, & \text { if } v_{j, i}=\theta_{i, \min }
\end{array}\right. \\
& b_{i_{\min , j}}=\left\{\begin{array}{cl}
\frac{1}{\theta_{i, \max }-\theta_{i, \min },} & \text { if } v_{j, i}=\theta_{i, \min } \\
0, & \text { if } v_{j, i}=\theta_{i, \max } .
\end{array}\right.
\end{aligned}
$$

The following steps are then carried out in every sampling instant:

$$
\begin{aligned}
& \text { 1. } \theta_{i, k}=\cos \left(2 \pi f_{i, k} T\right), i=1, \ldots, N, \\
& \text { 2. } c_{i_{\max }, k}=\theta_{i, k}-\theta_{i, \min }, \quad c_{i_{\min }, k}=\theta_{i, \max }-\theta_{i, k}, i=1, \ldots, N, \\
& \text { 3. } \lambda_{j, k}=\prod_{i=1}^{N}\left(b_{i_{\max }, j} c_{i_{\max }, k}+b_{i_{\min }, j} c_{i_{\min }, k}\right), j=1, \ldots, M .
\end{aligned}
$$

\section{Application for the rejection of harmonic disturbances}

In this section, the methods described in Sec. 4 are applied to the case of a harmonic multisine disturbance. Specific system models and the transformation that leads to a pLPV system are presented.

\subsection{Internal model for harmonic disturbances}

The disturbance is assumed to be a multisine. Let $N$ be the number of components of the disturbance and $f_{i, k}$ the frequency of the $i$-th component at sampling instant $k$. The frequencies are assumed to vary in intervals $\left[f_{i, \min }, f_{i, \max }\right] \subset\left[0,0.5 f_{\mathrm{s}}\right]$, where $f_{\mathrm{s}}$ denotes the sampling frequency.

As discussed in Sec. 3, an error filter or a disturbance model can be used to include desired dynamics in the controller according to the internal model principle [14]. Since the following relations are used for either $A_{\mathrm{M}, k}$ or $A_{\mathrm{d}, k}$ depending on the control approach, the notation $A_{\mathrm{M} / \mathrm{d}, k}$ is employed. The dynamics for a multisine disturbance with time-varying frequency are given by a discrete-time state-space model with system matrix

$$
A_{\mathrm{M} / \mathrm{d}, k}=\left[\begin{array}{ccc}
A_{\mathrm{M}_{1} / \mathrm{d}_{1}, k} & \cdots & \mathbf{0} \\
\vdots & \ddots & \vdots \\
\mathbf{0} & \cdots & A_{\mathrm{M}_{N} / \mathrm{d}_{N}, k}
\end{array}\right]
$$


where

$$
A_{\mathrm{M}_{i} / \mathrm{d}_{i, k}}=\left[\begin{array}{cc}
\cos \left(2 \pi f_{i, k} T\right) & \sin \left(2 \pi f_{i, k} T\right) \\
-\sin \left(2 \pi f_{i, k} T\right) & \cos \left(2 \pi f_{i, k} T\right)
\end{array}\right]
$$

with sampling time $T=1 / f_{\mathrm{s}}$. This time-varying matrix might therefore be used as system matrix $A_{\mathrm{M}, k}$ of the error filter described in Sec. 3.1 or $A_{\mathrm{d}, k}$ of the disturbance model described in Sec. 3.2. In order to find a representation of $A_{\mathrm{M} / \mathrm{d}, k}$ that depends affinely on a parameter vector $\boldsymbol{\theta}$, one approach could be to choose

$$
\theta_{i, k}=\cos \left(2 \pi f_{i, k} T\right), i=1, \ldots, N .
$$

Unfortunately, through

$$
\sin \left(2 \pi f_{i, k} T\right)=\sqrt{1-\theta_{i, k}^{2}}, i=1, \ldots, N,
$$

a non-affine dependence is introduced into the model. To circumvent this, additional parameters

$$
\theta_{i, k}=\sin \left(2 \pi f_{i, k} T\right), i=N+1, \ldots, 2 N
$$

could be introduced. The use of this internal model leads to a large dimension of the parameter space and a polytope with many vertices. It can be expected that controllers based on this model are quite conservative, if there exists a solution to the LMIs at all. Therefore, a simplified disturbance model that leads to less conservative conditions is used for the controller design.

In the case of constant frequencies, instead of (84), the matrices

$$
A_{\mathrm{M}_{i} / \mathrm{d}_{i}}=\left[\begin{array}{cc}
0 & 1 \\
-1 & 2 \cos \left(2 \pi f_{i}, T\right)
\end{array}\right], i=1, \ldots, N
$$

can be used in the system matrix of the internal model (83). This leads to a time-invariant overall system model. As discussed in Sec. 3, the internal model then is a way to determine controller poles (that show up as zeros in the disturbance transfer functions). With the above approach, for a frequency $f$, a complex conjugate controller pole pair is placed at $\exp (j 2 \pi f T)$ and $\exp (-j 2 \pi f T)$, which causes complete asymptotic rejection of harmonic disturbances with this frequency.

Although this model does not take into account the rate of change of the disturbance frequencies and the argumentation based on controller poles is valid only in the time-invariant case, this simpler model is used here even for the case of time-varying frequencies, since it reduces the dimension of the parameter space. Thus, the matrices

$$
A_{\mathrm{M}_{i} / \mathrm{d}_{i}, k}=\left[\begin{array}{cc}
0 & 1 \\
-1 & 2 \cos \left(2 \pi f_{i, k} T\right)
\end{array}\right], i=1, \ldots, N
$$

are used for the time-varying disturbance model. This might have an effect on the disturbance attenuation for fast changes of the frequencies, but it is expected that other effects are more dominant than the "incorrect" disturbance model. There will always be delay between the measured frequency used in the controller and the true frequency. Also, if the disturbance and the control signal do not enter at exactly the same point and the disturbance frequency varies, it is unclear which frequency "is present" at the point where the control signal enters 
the plant at a certain time. As shown in Sec. 3, closed-loop stability is not affected by the choice of the internal model.

\subsection{Transformation to pLPV form}

If the internal model can be written in the form of a pLPV system, it is possible to find a representation of the system matrix $A_{k}$ of the overall system of the error filter approach (5) and the disturbance observer approach (15) in the form of a pLPV system as well. Then, the controller design and the gain-scheduling procedure can be carried out based on quadratic stability theory of pLPV systems, as has been presented in Sec. 4.

If the sampling theorem applies (which should be the case for any practical application), it holds that $0 \leq 2 \pi f_{i, k} T \leq \pi$ for all $i=1, \ldots, N$. Since the function $f \mapsto \cos (2 \pi f T)$ is monotonically decreasing on $[0, \pi]$, the frequencies under consideration have to fulfill $0 \leq 2 \pi f_{i, k} T \leq \pi$ for all $i=1, \ldots, N$ in order to guarantee a unique mapping between frequencies and parameters. This can usually be achieved by choosing a sufficiently small value for $T$. Then, parameters $\boldsymbol{\theta}_{i}$ as defined in (85) are bounded by

$$
\theta_{i, \min }=\cos \left(2 \pi f_{i, \max } T\right), \theta_{i, \max }=\cos \left(2 \pi f_{i, \min } T\right), i=1, \ldots, N,
$$

respectively. Defining

$$
\boldsymbol{\Theta}=\left[\theta_{1, \min }, \theta_{1, \max }\right] \times\left[\theta_{2, \min }, \theta_{2, \max }\right] \times \ldots \times\left[\theta_{N, \min }, \theta_{N, \max }\right] \subset \mathbb{R}^{N}
$$

with vertices $v_{1}, v_{2}, \ldots, v_{M} \in \mathbb{R}^{N}$ it follows that the parameter vector

$$
\boldsymbol{\theta}_{k}=\left[\begin{array}{c}
\theta_{1, k} \\
\theta_{2, k} \\
\vdots \\
\theta_{N, k}
\end{array}\right] \in \mathbb{R}^{N}
$$

is always inside the hyper box $\Theta$. Then, the system matrix $A_{\mathrm{M} / \mathrm{d}, k}$ of the internal model can be written as

$$
A_{\mathrm{M} / \mathrm{d}, k}=\boldsymbol{A}_{\mathrm{M} / \mathrm{d}}\left(\boldsymbol{\theta}_{k}\right)=\boldsymbol{A}_{\mathrm{M}_{0} / \mathrm{d}_{0}}+\theta_{1, k} \mathcal{A}_{\mathrm{M}_{1} / \mathrm{d}_{1}}+\theta_{2, k} \mathcal{A}_{\mathrm{M}_{2} / \mathrm{d}_{2}}+\ldots+\theta_{N, k} \mathcal{A}_{\mathrm{M}_{N} / \mathrm{d}_{N}}
$$

with

$$
\mathcal{A}_{\mathrm{M}_{0} / \mathrm{d}_{0}}=\left[\begin{array}{ccc}
\mathcal{A}_{\mathrm{M}_{0} / \mathrm{d}_{0}, 1} & \cdots & \mathbf{0} \\
\vdots & \ddots & \vdots \\
\mathbf{0} & \cdots & \mathcal{A}_{\mathrm{M}_{0} / \mathrm{d}_{0}, N}
\end{array}\right]
$$

where

$$
\mathcal{A}_{\mathrm{M}_{0} / \mathrm{d}_{0}, i}=\left[\begin{array}{cc}
0 & 1 \\
-1 & 0
\end{array}\right], i=1, \ldots, N,
$$

and $\mathcal{A}_{\mathrm{M}_{i} / \mathrm{d}_{i}}$ matrices with zero entries only except for

$$
\mathcal{A}_{\mathrm{M}_{i} / \mathrm{d}_{i}}(2 i, 2 i)=2, i=1, \ldots, N .
$$

Therefore, the system matrix $A_{\mathrm{M} / \mathrm{d}, k}$ of the internal model used for the error filter from Sec. 3.1 and the disturbance model in Sec. 3.2 can be written in pLPV form based on the 
$N$-dimensional hyper box $\Theta$ with vertices $v_{1}, v_{2}, \ldots, v_{M} \in \mathbb{R}^{N}$. The system matrices $A_{k}$ of (5) in the error filter approach can then be written as

$$
\boldsymbol{A}_{k}=\boldsymbol{A}\left(\boldsymbol{\theta}_{k}\right)=\boldsymbol{\mathcal { A }}_{0}+\theta_{1, k} \mathcal{A}_{1}+\ldots+\theta_{N, k} \mathcal{A}_{N}
$$

with

$$
\begin{gathered}
\mathcal{A}_{0}=\left[\begin{array}{cc}
\mathcal{A}_{\mathrm{M}_{0}} & \boldsymbol{B}_{\mathrm{M}} C_{\mathrm{p}} \\
\mathbf{0} & A_{\mathrm{p}}
\end{array}\right], \\
\mathcal{A}_{i}=\left[\begin{array}{cc}
\mathcal{A}_{\mathrm{M}_{i}} & 0 \\
\mathbf{0} & \mathbf{0}
\end{array}\right], i=1, \ldots, N,
\end{gathered}
$$

and analogously in (15) for the disturbance observer approach

$$
\boldsymbol{A}_{k}=\boldsymbol{A}\left(\boldsymbol{\theta}_{k}\right)=\mathcal{A}_{0}+\theta_{1, k} \mathcal{A}_{1}+\ldots+\theta_{N, k} \mathcal{A}_{N}
$$

with

$$
\begin{gathered}
\mathcal{A}_{0}=\left[\begin{array}{cc}
A_{\mathrm{p}} & \boldsymbol{B}_{\mathrm{p}} C_{\mathrm{d}} \\
\mathbf{0} & \mathcal{A}_{\mathrm{d}_{0}}
\end{array}\right], \\
\mathcal{A}_{i}=\left[\begin{array}{cc}
\mathbf{0} & \mathbf{0} \\
\mathbf{0} & \mathcal{A}_{\mathrm{d}_{i}}
\end{array}\right], i=1, \ldots, N .
\end{gathered}
$$

Since $\Theta$ is a convex polytope, in every instant there exist coordinates $\lambda_{j, k}, j=1, \ldots, M$, such that

$$
\boldsymbol{\theta}_{k}=\sum_{j=1}^{M} \lambda_{j, k} \boldsymbol{v}_{j} .
$$

Since $A_{k}$ depends affinely on $\boldsymbol{\theta}_{k}$ in both approaches, it follows that the system matrix can be expressed as

$$
\boldsymbol{A}\left(\boldsymbol{\theta}_{k}\right)=\sum_{j=1}^{M} \lambda_{j, k} \boldsymbol{A}_{\mathrm{v}, j}=\boldsymbol{A}\left(\lambda_{k}\right),
$$

where

$$
A_{\mathrm{v}, j}=\boldsymbol{A}\left(\boldsymbol{v}_{j}\right), j=1, \ldots, M .
$$

The required time-varying state-feedback and observer gains for the overall systems (5) and (15), respectively, can therefore be obtained by application of the design methods based on quadratic stability theory as reviewed in Sec. 4.

\subsection{Controller implementation}

Once the pLPV representation of the considered system is found, the required time-varying state-feedback and observer gains for the overall systems (5) and (15), respectively, can be obtained by application of the design methods based on quadratic stability theory as reviewed in Sec. 4.

The time-invariant vertex state-feedback or vertex observer gains are calculated in advance. Since the plant itself is linear time-invariant, also the time-invariant observer required for the estimation of the plant states in the error filter approach as well as the time-invariant state-feedback gain of the disturbance observer approach can be calculated off-line with 


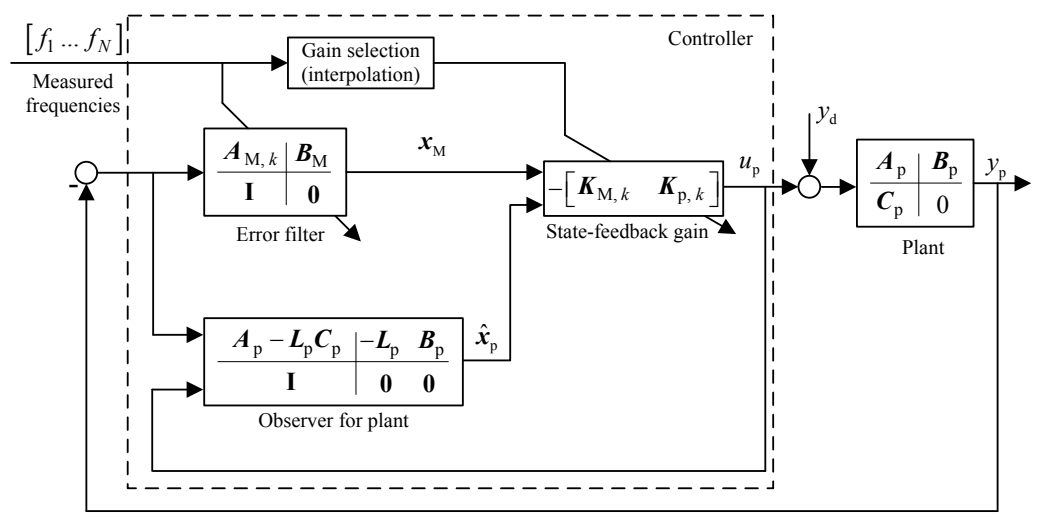

Figure 8. Control structure and interpolation for the error filter approach

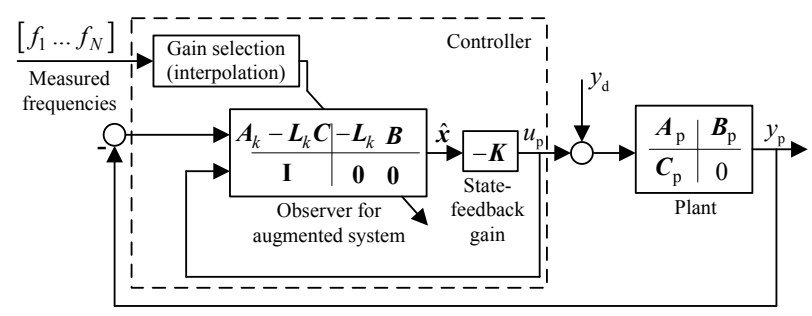

Figure 9. Control structure and interpolation the disturbance model approach

a standard method (e.g. LQR or pole placement). The controllers are then implemented according to the structures given in Sec. 3 and shown in Figs. 8 and 9 with the interpolation. Wherever possible, system matrices are updated in every sampling instant directly with the measured frequencies, while the time-varying state-feedback and observer gains are obtained by interpolation with the coordinates obtained by the scheme given in Sec. 4.4.

\section{Real-time results}

The gain-scheduled observer-based state-feedback controllers obtained through the design procedures presented in this chapter are validated with experimental results. Both controllers have been tested on an AVC and an ANC system and were found to work well. Results are presented for the test of the error filter with time-varying state-feedback gain on an ANC headset and the controller based on a disturbance observer with time-varying observer gain on an AVC test bed.

\subsection{Real-time results for state-feedback gain-scheduling (error filtering) approach}

The controller based on a disturbance observer with time-varying observer gain is tested experimentally on a Sennheiser PXC 300 headset. The experimental setup is shown schematically in Fig. 10. An external loudspeaker is used to generate a harmonic disturbance. The headset has one microphone on each loudspeaker. The objective is to cancel the 


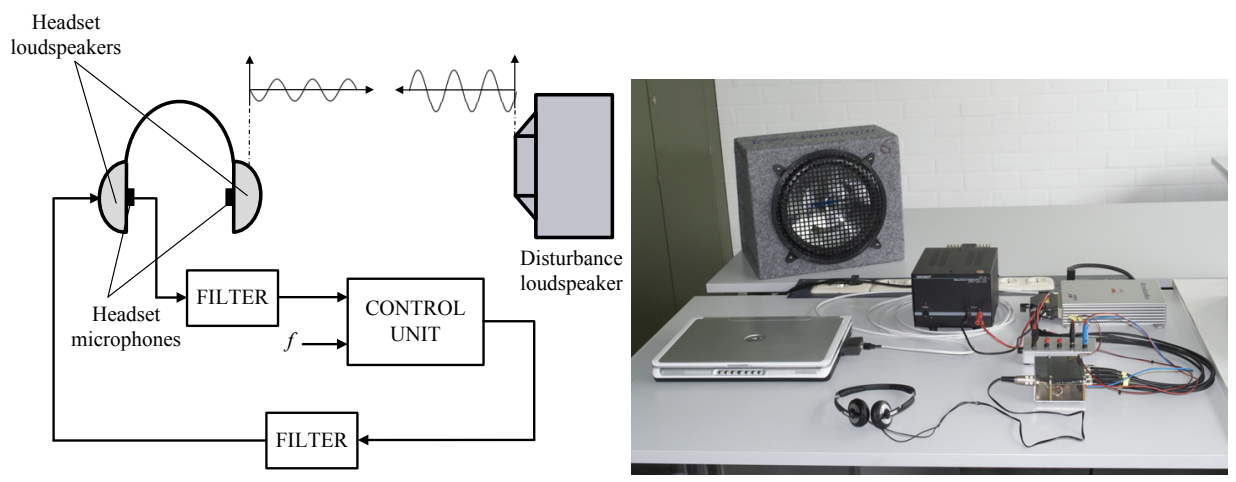

Figure 10. Block diagramm (left) and photograph (right) of the ANC system

disturbance with the loudspeakers of the headset. An anti-aliasing filter is applied to the output signal and a reconstruction filter to the control input. The control algorithm is implemented on a rapid control prototyping unit (dSpace MicroAutoBox). A sampling frequency of $1 \mathrm{kHz}$ was used.

Standard black-box system identification techniques were used to obtain the transfer function between output and input of the control unit. The identified transfer function is of 12th order and the resulting controller of 20th order. As a disturbance signal, a sum of four harmonically related sine signals with fundamental frequency between $90 \mathrm{~Hz}$ and $100 \mathrm{~Hz}$ is used. The control algorithm has been implemented on both sides and results for the right side are shown.

Fig. 11 shows the amplitude frequency responses for constant fundamental frequencies of $90 \mathrm{~Hz}$ and $100 \mathrm{~Hz}$. The amplitude frequency response plots show that amplification takes place in some frequency ranges, which is the known "waterbed" effect. It might cause problems in practical applications where significant background noise, e.g. broadband stochastic disturbances, is present. In Fig. 12, results are shown for a case where the fundamental frequency suddenly jumps from $90 \mathrm{~Hz}$ to $100 \mathrm{~Hz}$. The controller remains stable even for such drastic variations of the fundamental frequency. The transient spike might be undesirable, particularly in ANC applications where it could be audible. However, a step change in the frequency does not commonly occur in real applications.

In Fig. 13, results for a gradually changing fundamental frequency are shown. The measurements show that excellent disturbance attenuation is achieved. The performance of the controller is tested with fast variations of the disturbance frequencies. Results for this experiment are shown in Fig. 14. It can be observed that the attenuation performance decreases, but the system remains stable.

\subsection{Real-time results for observer gain-scheduling (disturbance model) approach}

The scheme and a photograph of the AVC test bed are shown in Fig. 15. Two shakers (inertia mass actuators) are attached to a steel cantilever beam. One shaker acts as the disturbance source and the other shaker is driven by the control signal to counteract this disturbance. An accelerometer is used to measure the output signal. An anti-aliasing filter is applied to the output signal and a reconstruction filter to the control input. 

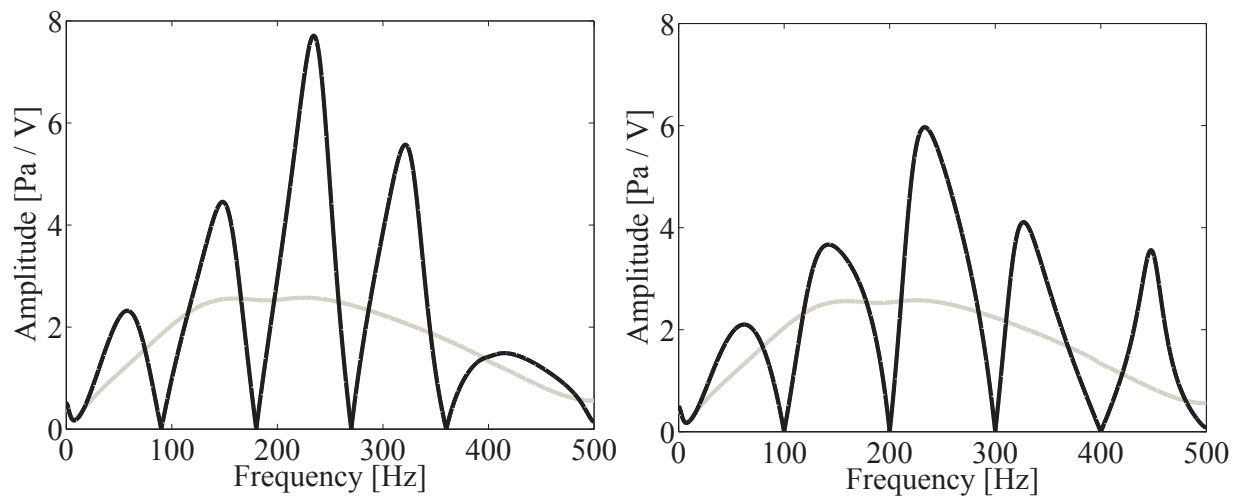

Figure 11. Open-loop (gray) and closed-loop (black) amplitude frequency responses for fixed disturbance frequencies of 90, 180, 270 and $360 \mathrm{~Hz}$ (left) and of 100, 200, 300 and $400 \mathrm{~Hz}$ (right)
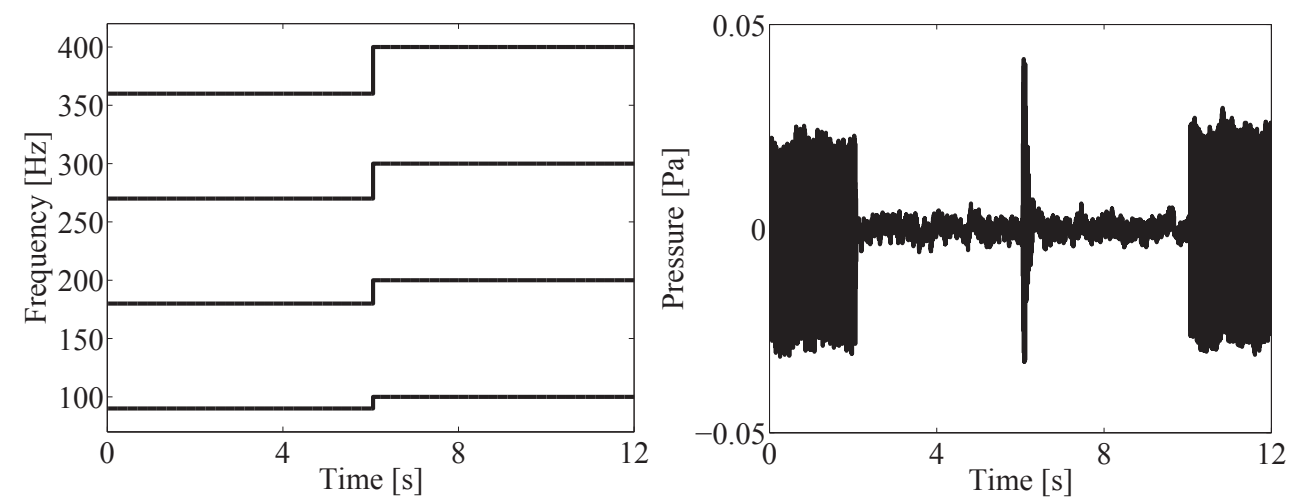

Figure 12. Results for a disturbance with time-varying frequencies. Variation of the frequencies (left) and measured sound pressure (right). The control sequence is off/on/off

A multisine test signal is used to identify the transfer function between output and input of the control unit using standard black-box system identification techniques. The controller is implemented on a rapid control prototyping unit (dSpace MicroAutoBox). A sampling frequency of $1 \mathrm{kHz}$ was chosen. The identified system is of 10th order and the controller of 16th order. As a disturbance signal, a sum of three harmonically related sine signals with fundamental frequency between 110 and $120 \mathrm{~Hz}$ is used. The amplitude frequency responses of the open-loop and closed-loop systems for fixed disturbance frequencies of $110 \mathrm{~Hz}, 220 \mathrm{~Hz}$ and $330 \mathrm{~Hz}$ and $120 \mathrm{~Hz}, 240 \mathrm{~Hz}$ and $360 \mathrm{~Hz}$ are shown in Fig. 16. The resonance frequencies are damped by a factor 0.9 using pole placement with the state-feedback gain $K_{\mathrm{p}}$. In Fig. 17, results are shown for a (rather unrealistic) case where the fundamental frequency suddenly jumps from $110 \mathrm{~Hz}$ to $120 \mathrm{~Hz}$. After a short transient spike the disturbance rejection resumes quickly. The transient spike might be undesirable but a step change in the frequency does not commonly occur in real applications. The measurements confirm the excellent disturbance rejection that can be expected from the amplitude frequency responses. The amplitude response plots show that amplification takes place in frequency ranges between the rejected 

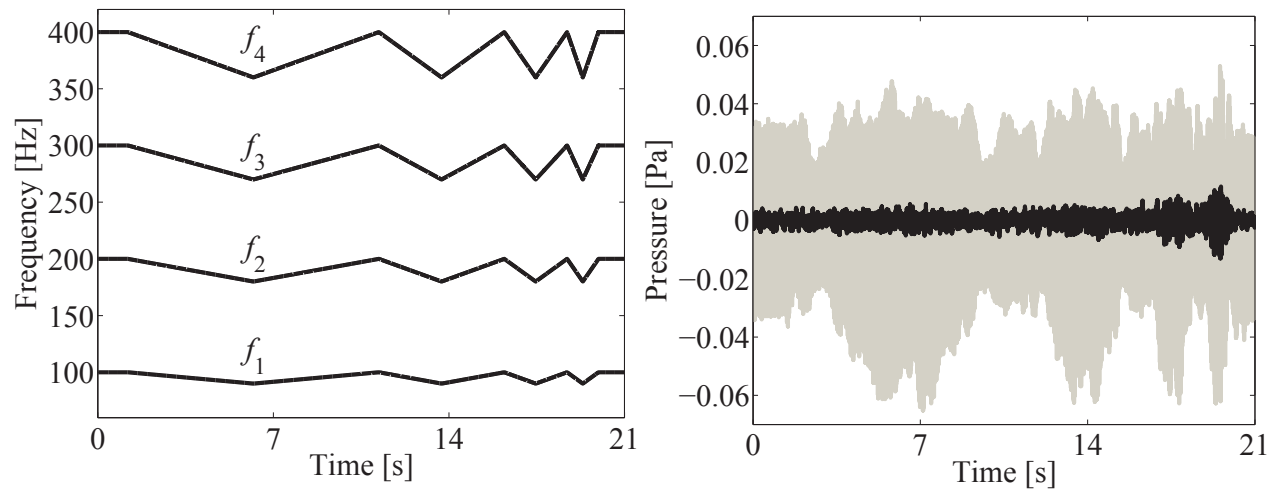

Figure 13. Results for a disturbance with time-varying frequencies. Variation of the frequencies (left) and measured sound pressure (right) in open loop (gray) and closed loop (black)
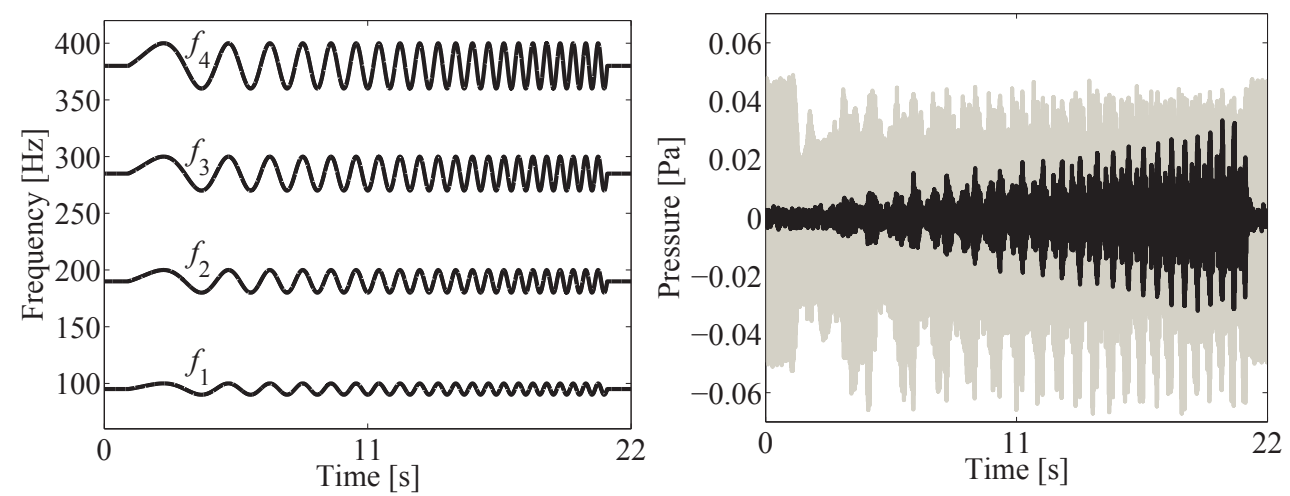

Figure 14. Results for a disturbance with time-varying frequencies. Variation of the frequencies (left) and measured sound pressure (right) in open loop (gray) and closed loop (black)
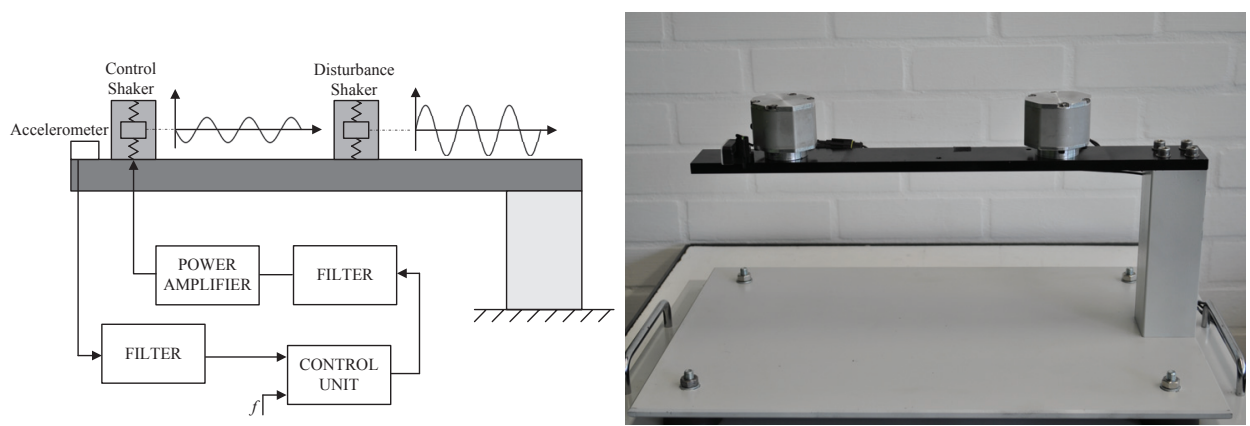

Figure 15. Schematic representation (left) and photograph (right) of the AVC system 
frequencies. This is due to Bode's sensitivity integral ("waterbed" effect). Whether this is tolerable or not depends on the application. It is possible to limit the maximum disturbance amplification by a suitable overall controller design (which need not be observer based), but at the expense of either not fully suppressing the harmonic disturbances (that is, the notches in the frequency response would not get to zero) or requiring the frequency measurements to be very exact (that is, making the notches narrower) or worsening the transient behavior (that is, it would take longer before a harmonic disturbance is suppressed to a certain level). However, in this chapter only harmonic disturbances are considered, so these issues are not addressed in the design.

In Fig. 18, results for a gradually changing fundamental frequency are shown. It decreases linearly from $120 \mathrm{~Hz}$ to $110 \mathrm{~Hz}$, remains constant for a while and then rises back to 120 $\mathrm{Hz}$. This is repeated four times, every time in a shorter time interval. At the end the fourth harmonic rises from $330 \mathrm{~Hz}$ to $360 \mathrm{~Hz}$ and decreases back to $330 \mathrm{~Hz}$ in less than two seconds. The effect of fast variations of the disturbance frequencies has been further investigated in another experiment. The results are shown in Fig. 19. The disturbance frequency varies
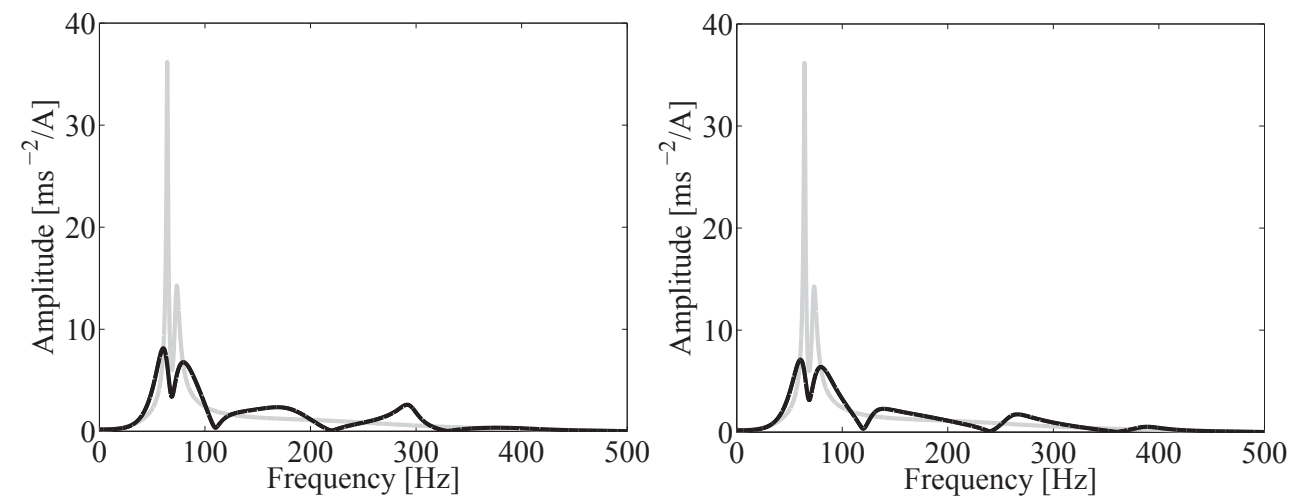

Figure 16. Open-loop (gray) and closed-loop (black) amplitude frequency responses for fixed disturbance frequencies of 110, 220 and $330 \mathrm{~Hz}$ (left) and of 120, 240 and $360 \mathrm{~Hz}$ (right)
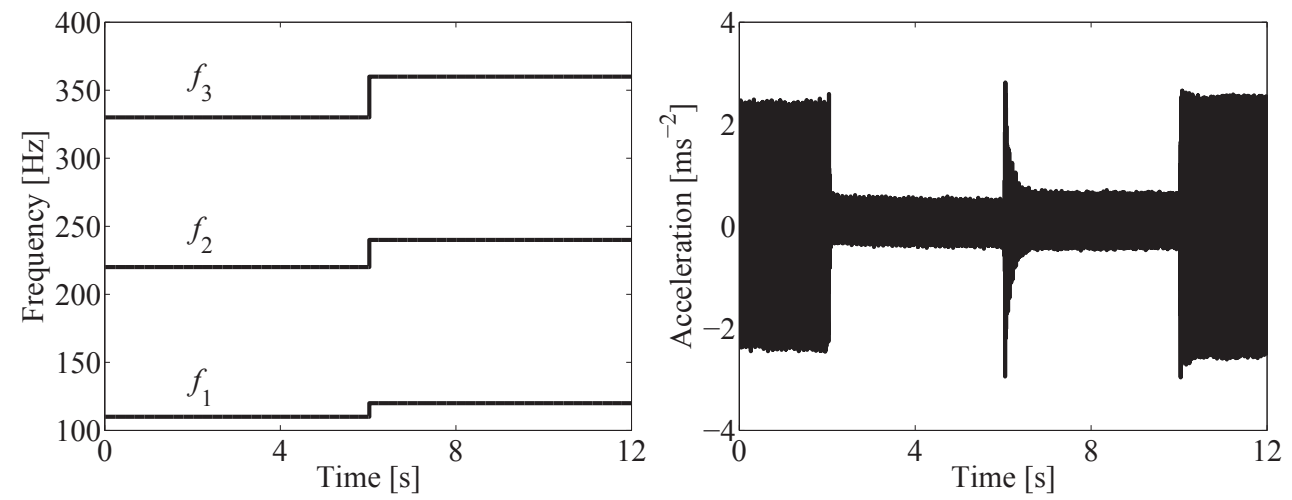

Figure 17. Results for a disturbance with time-varying frequencies. Variation of the frequencies (left) and measured acceleration (right). The control sequence is off/on/off 
sinusoidally between the minimum and the maximum value with a period that decays from 10 to 0.5 seconds. It is seen that for very fast frequency variations, the attenuation performance decreases slightly (but the system remains stable).
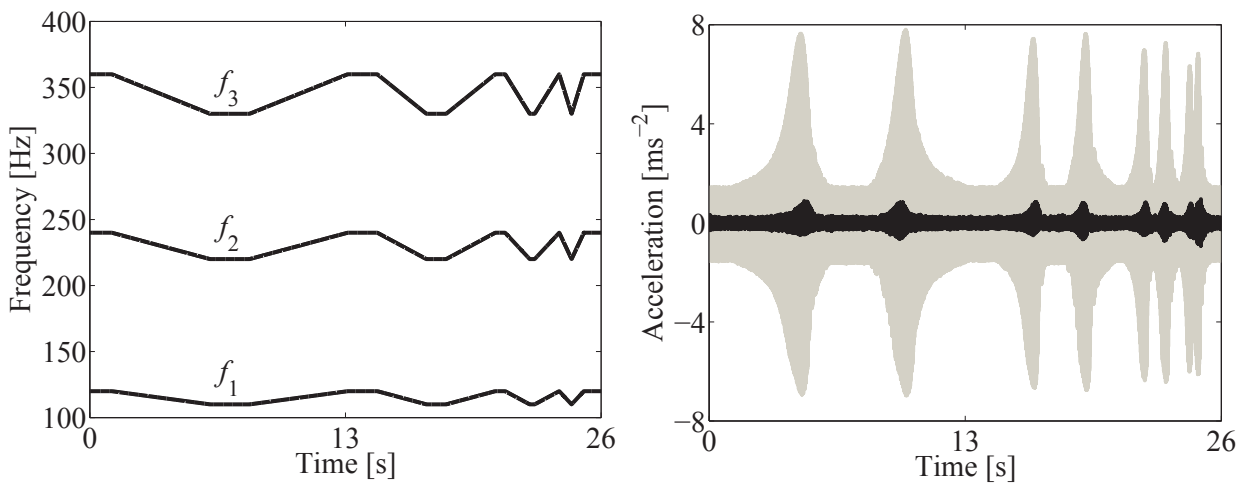

Figure 18. Results for a disturbance with time-varying frequencies. Variation of the frequencies (left) and measured acceleration (right) in open loop (gray) and closed loop (black)
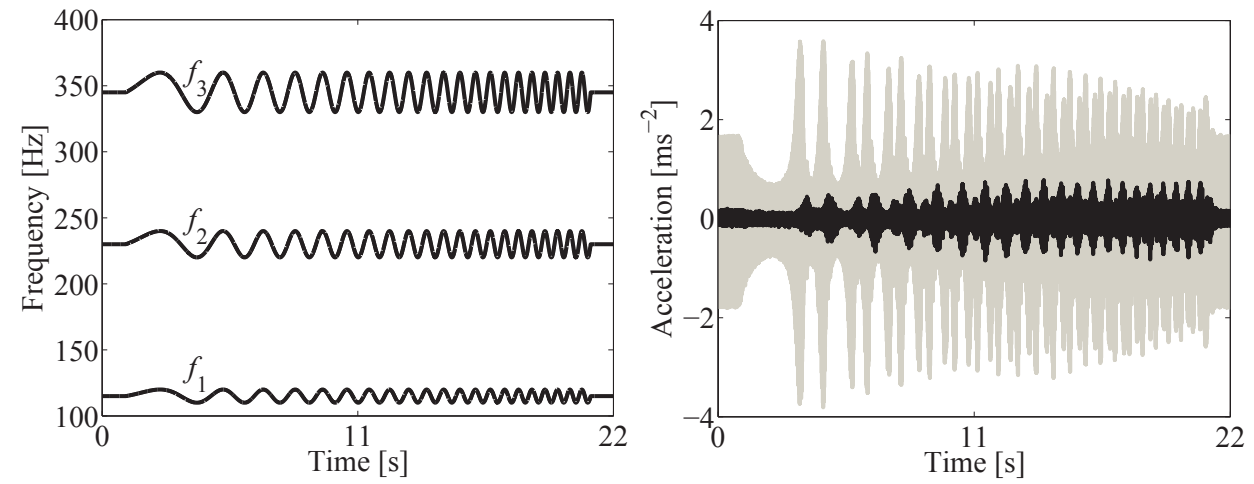

Figure 19. Results for a disturbance with time-varying frequencies. Variation of the frequencies (left) and measured acceleration (right) in open loop (gray) and closed loop (black)

\section{Discussion and conclusion}

Two discrete-time LPV observer-based state-feedback controllers for the rejection of harmonic disturbances with time-varying frequencies are presented. The control design methods are based on quadratic stability theory for pLPV systems. They guarantee stability of the closed-loop system also for arbitrarily fast changes of the disturbance frequencies. This is an advantage over other approaches such as adaptive filtering or heuristic gain scheduling. The experimental results show that an excellent disturbance rejection is achieved and that the proposed controllers can be applied in a real-time setup. 
However, some degree of conservatism is present in these approaches. Using a single parameter independent Lyapunov function limits the range of admissible disturbance frequencies that can be covered with the resulting controller. Also, the polytope that contains the uncertain parameters could be chosen much smaller and with fewer vertices than the cuboid applied here, if information on the relations between the disturbance frequencies is given (as, for example, in the case of harmonically related frequencies). Thus, feasibility of the LMIs and the upper bound on the system performance could be improved as well as the computation time for the coordinates required for the on-line interpolation. This might be important for applications where many harmonics have to be cancelled, a wide frequency range has to be covered and the computational resources are limited, for example in automotive applications [7]. This aspect as well as a direct comparison of the proposed methods with conventional algorithms (such as the FxLMS) will be subject of future research.

To the best of the authors' knowledge, industrial applications of LPV controllers are rather limited. The results of this and the following chapter show that the implementation of even high-order LPV controllers can be quite straightforward.

\section{Nomenclature}

Acronyms

ANC Active noise control.

AVC Active vibration control.

FxLMS Filtered-x least mean squares.

LFT Linear fractional transformation.

LMI Linear matrix inequality.

LPV Linear parameter varying.

pLPV Polytopic linear parameter varying.

Variables

(in order of appearance)

$A_{\mathrm{p}}, B_{\mathrm{p}}, C_{\mathrm{p}}$ State-space matrices of the plant.

$x_{\mathrm{p}, k} \quad$ State vector of the plant.

$y_{\mathrm{p}, k}, u_{\mathrm{p}, k}$ Output signal and control input of the plant.

$y_{\mathrm{d}, k} \quad$ Disturbance signal.

$A_{\mathrm{M}}, B_{\mathrm{M}} \quad$ State-space matrices of the error filter.

$x_{\mathrm{M}, k} \quad$ State-space vector of the error filter.

$r_{k}, e_{k} \quad$ Reference and error signal.

$A_{\mathrm{M}, k} \quad$ Time-varying system matrix of the error filter. 


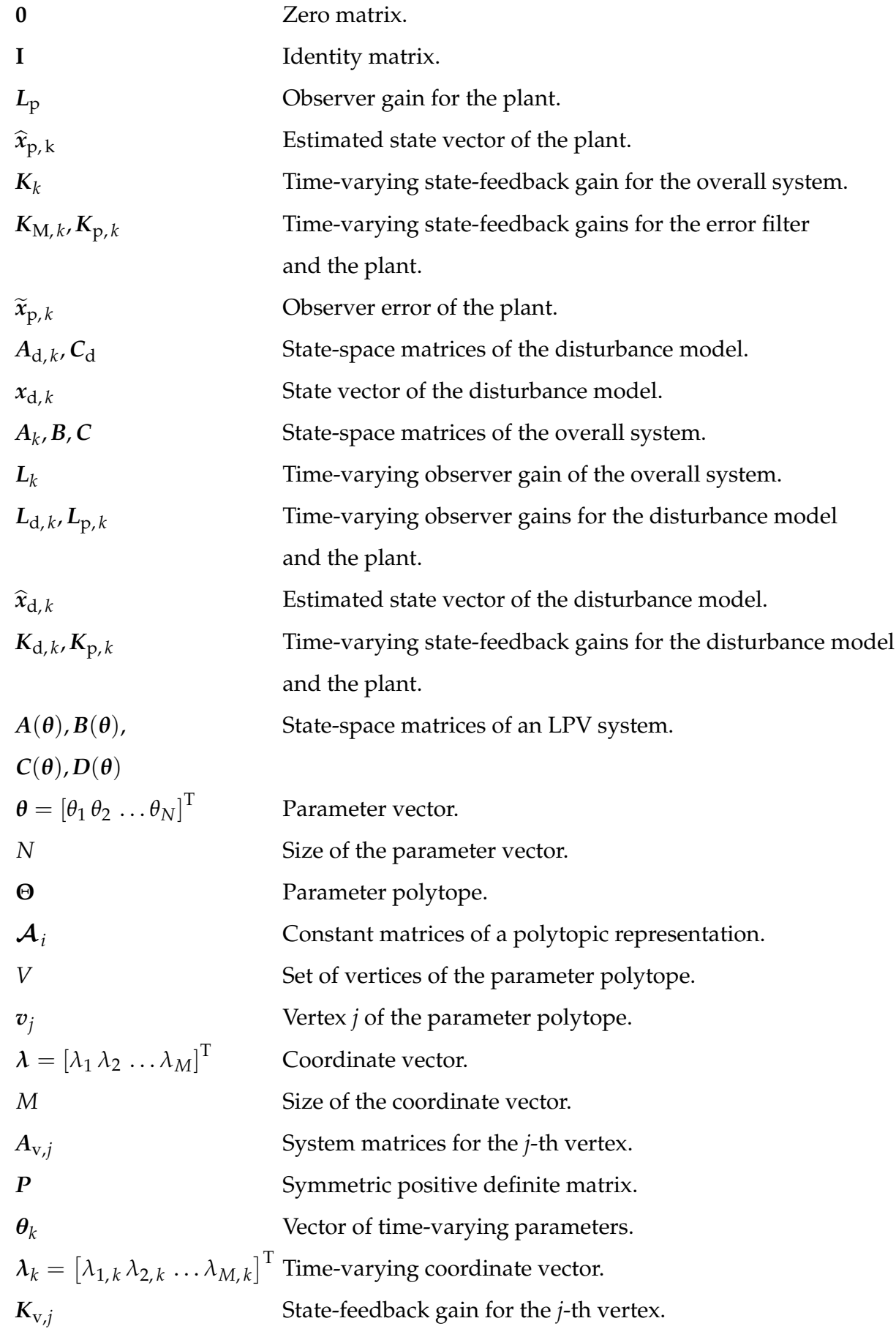


$\boldsymbol{w}_{k}, \boldsymbol{z}_{k}, \boldsymbol{u}_{k} \quad$ Process noise, performance output and control input.

$Q, R \quad$ Weighting matrices.

$G \quad$ Transfer function between $w_{k}$ and $z_{k}$.

$\widetilde{A}, \widetilde{B}, \widetilde{C} \quad$ State-space matrices of the transfer path from $w_{k}$ to $z_{k}$.

$\widetilde{Q}, \widetilde{R} \quad$ Matrices to build the LMIs.

$W_{\mathrm{c}} \quad$ Controllability gramian.

$\boldsymbol{W}_{\mathrm{O}} \quad$ Observability gramian.

W Symmetric positive definite matrix.

$Y_{\mathrm{v}, j} \quad$ Solution of the LMIs for the $j$-th vertex.

$L_{\mathrm{v}, j} \quad$ Observer gain for the $j$-th vertex.

$\boldsymbol{b}_{i_{\min }}, \boldsymbol{b}_{i_{\max }} \quad$ Vectors for the computation of the coordinate vector.

$c_{i_{\min }, k}, c_{i_{\max }, k}$, Scalars for the computation of the coordinate vector.

$b_{i_{\min }, j}, b_{i_{\max }, j}$

$A_{\mathrm{M} / \mathrm{d}, k} \quad$ System matrix of a general time-varying internal disturbance model.

\section{Author details}

Pablo Ballesteros, Christian Bohn, Wiebke Heins and Xinyu Shu

Institute of Electrical Information Technology, Clausthal University of Technology,

Clausthal-Zellerfeld, Germany

\section{References}

[1] Amato, F. 2006. Robust control of linear systems subject to uncertain time-varying parameters. Berlin: Springer.

[2] Apkarian, P., P. Gahinet and G. Becker. 1995. Self-scheduled control of linear parameter-varying systems: A design example. Automatica 31:1251-61.

[3] Apkarian, P. 1997. On the discretization of LMI-synthesized linear parameter-varying controllers. Automatica 33:655-61.

[4] Ballesteros, P. and C. Bohn. 2011a. A frequency-tunable LPV controller for narrowband active noise and vibration control. Proceedings of the American Control Conference. San Francisco, June 2011. 1340-45.

[5] Ballesteros, P. and C. Bohn. 2011b. Disturbance rejection through LPV gain-scheduling control with application to active noise cancellation. Proceedings of the IFAC World Congress. Milan, August 2011. 7897-902.

[6] Balini, H. M. N. K., C. W. Scherer and J. Witte. 2011. Performance enhancement for AMB systems using unstable $H_{\infty}$ controllers. IEEE Transactions on Control Systems Technology 19:1479-92.

[7] Bohn, C., A. Cortabarria, V. Härtel and K. Kowalczyk. 2003. Disturbance-observer-based active control of engine-induced vibrations in automotive vehicles. Proceedings of the 
SPIE's 10th Annual International Symposium on Smart Structures and Materials. San Diego, March 2003. Paper No. 5049-68.

[8] Bohn, C., A. Cortabarria, V. Härtel and K. Kowalczyk. 2004. Active control of engine-induced vibrations in automotive vehicles using disturbance observer gain scheduling. Control Engineering Practice 12:1029-39.

[9] Daafouz, J., G. I. Bara, F. Kratz and J. Ragot. 2000: State observers for discrete-time LPV systems: an interpolation based approach. Proceedings of the 39th IEEE Conference on Decision and Control 5: 4571-72.

[10] Darengosse C. and P. Chevrel. 2000. Linear parameter-varying controller design for active power filters. Proceedings of the IFAC Control Systems Design. Bratislava, June 2000. 65-70.

[11] $\mathrm{Du}, \mathrm{H}$. and X. Shi. 2002. Gain-scheduled control for use in vibration suppression of system with harmonic excitation. Proceedings of the American Control Conference. Anchorage, May 2002. 4668-69.

[12] Du, H., L. Zhang and X. Shi. 2003. LPV technique for the rejection of sinusoidal disturbance with time-varying frequency. IEE Proceedings on Control Theory and Applications 150:132-38.

[13] Feintuch, P. L., N. J. Bershad and A. K. Lo. 1993. A frequency-domain model for filtered LMS algorithms - Stability analysis, design, and elimination of the training mode. IEEE Transactions on Signal Processing 41:1518-31.

[14] Francis, B. and W. Wonham. 1976. The internal model principle of control theory. Automatica 12:457-65.

[15] Gahinet, P., A. Nemirovskii, A. J. Laub and M. Chilali. 1995. LMI Control Toolbox. The Mathworks Inc.

[16] Heins, W., P. Ballesteros and C. Bohn. 2011. Gain-scheduled state-feedback control for active cancellation of multisine disturbances with time-varying frequencies. Presented at the 10th MARDiH Conference on Active Noise and Vibration Control Methods. Krakow-Wojanow, Poland, June 2011.

[17] Heins, W., P. Ballesteros and C. Bohn. 2012. Experimental evaluation of an LPV-gain-scheduled observer for rejecting multisine disturbances with time-varying frequencies. Proceedings of the American Control Conference. Montreal, June 2012. Accepted for publication.

[18] Horn, R. A. and C. R. Johnson. 1985. Matrix Analysis. Cambridge: Cambridge UP.

[19] Kinney, C. E. and R. A. de Callafon. 2006a. Scheduling control for periodic disturbance attenuation. Proceedings of the American Control Conference. Minneapolis, June 2006. 4788-93.

[20] Kinney, C. E. and R. A. de Callafon. 2006b. An adaptive internal model-based controller for periodic disturbance rejection. Proceedings of the 14th IFAC Symposium on System Identification. Newcastle, Australia, March 2006. 273-78.

[21] Kinney, C. E. and R. A. de Callafon. 2007. A comparison of fixed point designs and time-varying observers for scheduling repetitive controllers. Proceedings of the 46 th IEEE Conference on Decision and Control. New Orleans, December 2007. 2844-49.

[22] Köroğlu , H. and C. W. Scherer. 2008. Robust generalized asymptotic regulation against non-stationary sinusoidal disturbances. Proceedings of the 47th IEEE Conference on Decision and Control. Cancun, December 2008. 5426-31. 
[23] Köroğlu, H. and C. W. Scherer. 2008. LPV control for robust attenuation of non-stationary sinusoidal disturbances with measurable frequencies. Proceedings of the 17th IFAC World Congress. Korea, July 2008. 4928-33.

[24] Kuo, S. M. and D. R. Morgan. 1996. Active Noise Control Systems. New York: Wiley.

[25] Morari, M. and E. Zafiriou 1989. Robust Process Control. Englewood Cliffs: Prentice Hall.

[26] Shu, X., P. Ballesteros and C. Bohn. 2011. Active vibration control for harmonic disturbances with time-varying frequencies through LPV gain scheduling. Proceedings of the 23rd Chinese Control and Decision Conference. Mianyang, China, May 2011. 728-33.

[27] Stilwell, D. J. and W. J. Rugh. 1998. Interpolation of observer state feedback controllers for gain scheduling. Proceedings of the American Control Conference 2:1215-19.

[28] Witte, J., H. M. N. K. Balini and C. W. Scherer. 2010. Experimental results with stable and unstable LPV controllers for active magnetic bearing systems. Proceedings of the IEEE International Conference on Control Applications. Yokohama, September 2010. 950-55. 